\title{
Symmetries and Exact Solutions of a 2+1-dimensional Sine-Gordon System
}

\author{
By Peter A. Clarkson, Elizabeth L. Mansfield and Alice E. Milne \\ Department of Mathematics, University of Exeter, Exeter, EX4 4QE, U.K.
}

\begin{abstract}
We investigate the classical and nonclassical reductions of the 2+1-dimensional sine-Gordon system of Konopelchenko and Rogers, which is a strong generalisation of the sine-Gordon equation. A family of solutions obtained as a nonclassical reduction involves a decoupled sum of solutions of a generalised, real, pumped Maxwell-Bloch system. This implies the existence of families of solutions, all occurring as a decoupled sum, expressible in terms of the second, third and fifth Painlevé transcendents, and the sine-Gordon equation. Indeed, hierarchies of such solutions are found, and explicit transformations connecting members of each hierarchy are given.

By applying a known Bäcklund transformation for the system to the new solutions found, we obtain further families of exact solutions, including some which are expressed as the argument and modulus of sums of products of Bessel functions with arbitrary coefficients.

Finally, we prove the sine-Gordon system has the Painlevé property, which requires the usual test to be modified, and derive a non-isospectral Lax pair for the generalised, real, pumped MaxwellBloch system.
\end{abstract}

\section{Introduction}

There is much current interest in $2+1$-dimensional equations, i.e. equations with two spatial and one temporal variables, which are solvable by inverse scattering and thus are completely integrable (cf., Ablowitz \& Clarkson 1991; Konopelchenko 1993). Notable examples include the KadomtsevPetviashvili (KP), Davey-Stewartson (DS) and Nizhnik-Veselov-Novikov (NVN) equations. The DS and NVN equations are "strong" generalisations of the nonlinear Schrödinger equation and Korteweg-de Vries (KdV) equation respectively, since the spatial variables arise on an equal basis. In contrast, the KP equation is not a strong generalisation of the KdV equation. Further, the DS and NVN equations admit coherent structure solutions (Boiti et al. 1988; Athorne \& Nimmo 1991, respectively); these are exponentially decaying solutions, often referred to as dromions (Fokas \& Santini 1989, 1990).

Recently Konopelchenko \& Rogers $(1991,1993)$ have re-derived the system of equations

$$
\begin{aligned}
& \left(\frac{\Theta_{X T}}{\sin \Theta}\right)_{X}-\left(\frac{\Theta_{Y T}}{\sin \Theta}\right)_{Y}-\frac{\Theta_{Y} \Phi_{X T}-\Theta_{X} \Phi_{Y T}}{\sin ^{2} \Theta}=0 \\
& \left(\frac{\Phi_{X T}}{\sin \Theta}\right)_{X}-\left(\frac{\Phi_{Y T}}{\sin \Theta}\right)_{Y}-\frac{\Theta_{Y} \Theta_{X T}-\Theta_{X} \Theta_{Y T}}{\sin ^{2} \Theta}=0
\end{aligned}
$$

within the context of a novel class of $2+1$-dimensional equations generalising work on infinitesimal Bäcklund transformations due to Loewner (1952). The system (1.1) is a "strong" 2+1-dimensional generalisation of the sine-Gordon equation

$$
\theta_{z t}=\sin \theta
$$


since reducing it via $\Phi=0, \Theta(X, Y, t)=\theta(z, t)$, where $z=\mu_{1} X+\mu_{2} Y$, with $\mu_{1}$ and $\mu_{2}$ constants, not both zero, and $t=T$, yields (1.2), i.e., the reduction to the sine-Gordon equation occurs for either of the spatial variables.

The system (1.1) is also obtained as a special reduction of an integrable 2+1-dimensional Toda lattice scheme (Rogers 1993). An auto-Bäcklund transformation and some exact coherent structure solutions for this system are given in Konopelchenko et al. (1992); see also Konopelchenko (1993). Further coherent structure solutions for (1.1) are given by Schief (1992) and other solitonic solutions are given by Nimmo $(1992,1993)$.

The special case of (1.1) when $\Phi=\Theta$ is the $2+1$-dimensional sine-Gordon equation

$$
\left(\frac{\Theta_{X T}}{\sin \Theta}\right)_{X}-\left(\frac{\Theta_{Y T}}{\sin \Theta}\right)_{Y}-\frac{\Theta_{Y} \Theta_{X T}-\Theta_{X} \Theta_{Y T}}{\sin ^{2} \Theta}=0
$$

which was also written down by Konopelchenko \& Rogers (1991). Remarkably, the 2+1-dimensional sine-Gordon equation (1.3) appears in the work of Darboux on classical differential geometry, Darboux (1887-1896), as noted by Johnson et al. (1994).

We remark that (1.3) is not the "natural" $2+1$-dimensional generalisation of the sine-Gordon equation (1.2) given by

$$
\Theta_{T T}-\Theta_{X X}-\Theta_{Y Y}=\sin \Theta .
$$

In fact, there is considerable evidence suggesting that (1.4) is not completely integrable, i.e. solvable by inverse scattering (cf., Clarkson 1986a).

Making the gauge transformation

$$
\Phi_{x t}=2 v_{x t} \sin \Theta+\Theta_{x t} \cos \Theta, \quad \Phi_{y t}=-2 v_{y t} \sin \Theta-\Theta_{y t} \cos \Theta,
$$

with $x=\frac{1}{2}(X+Y), y=\frac{1}{2}(X-Y), t=T$ and $u(x, y, t)=\frac{1}{2} \Theta(X, Y, T)$ to (1.1) yields the following system of equations (see Konopelchenko \& Dubrovsky 1993; Nimmo 1993),

$$
\begin{aligned}
& \Delta_{1} \equiv u_{x y t}+u_{x} v_{y t}+u_{y} v_{x t}=0 \\
& \Delta_{2} \equiv v_{x y}-u_{x} u_{y}=0
\end{aligned}
$$

and it is this system which we study. The Lax pair for (1.6) is given by

$$
\begin{aligned}
\psi_{1, x}+u_{x} \psi_{2} & =0, \\
\psi_{2, y}-u_{y} \psi_{1} & =0, \\
\psi_{1, y t}+u_{y} \psi_{2, t}+v_{y t} \psi_{1} & =0, \\
\psi_{2, x t}-u_{x} \psi_{1, t}+v_{x t} \psi_{2} & =0,
\end{aligned}
$$

since $\psi_{1, x y t}=\psi_{1, y t x}$ and $\psi_{2, x y t}=\psi_{2, y t x}$ if and only if $u$ and $v$ satisfy (1.6). We remark that the spectral part of the Lax pair (1.7) is the same as that for the DSI equation, which was solved by inverse scattering by Ablowitz \& Haberman (1975) and Fokas \& Ablowitz (1984). By eliminating $v_{t}$ in $(1.6 a)$ one obtains,

$$
u_{x y t}+m_{1}(y, t) u_{x}+m_{2}(x, t) u_{y}+u_{x} \partial_{x}^{-1}\left(u_{x} u_{y}\right)_{t}+u_{y} \partial_{y}^{-1}\left(u_{x} u_{y}\right)_{t}=0,
$$

where $\partial_{x}^{-1} f(x)=\int_{-\infty}^{x} f\left(x_{1}\right) \mathrm{d} x_{1}, \partial_{y}^{-1} f(y)=\int_{-\infty}^{y} f\left(y_{1}\right) \mathrm{d} y_{1}$, and $m_{1}(y, t)=\lim _{x \rightarrow-\infty} v_{y}(x, y, t)$, $m_{2}(x, t)=\lim _{y \rightarrow-\infty} v_{x}(x, y, t)$ are functions of integration. The inverse scattering transform and initial value problem for this equation was studied by Konopelchenko \& Dubrovsky (1993; Dubrovsky \& Konopelchenko 1993) for the case when the boundary values $m_{1}$ and $m_{2}$ in (1.9) are constants.

In $\S \S 2$ and 3 of this paper, we consider reductions of the system (1.6) using the classical Lie method and the so-called "nonclassical method" due to Bluman \& Cole (1969), respectively. Using 
this nonclassical method, we obtain a family of solutions involving a decoupled sum of solutions of a real generalised Maxwell-Bloch system. This family includes as special cases solutions expressible in terms of solutions of the sine-Gordon equation (1.2) and the second, third and fifth Painlevé equations, which are discussed in $\S 4$. Indeed, hierarchies of such solutions are given, and the transformations connecting members of each hierarchy are given explicitly. In $\S 5$ we apply a known Bäcklund transformation (5.2) for the system (1.6) to our new exact solutions in some simple cases, to obtain further families of solutions including solutions expressed in terms of expansions of exponential or Bessel functions with arbitrary coefficients.

In $\S 6$, we demonstrate that the sine-Gordon system (1.6) has the Painlevé property due to Weiss et al. (1983); this requires a modification of the usual procedure given in Weiss et al. (1983). Further we discuss the integrability of the generalised Maxwell-Bloch system which we obtained as a nonclassical reduction. Finally in $\S 7$ we briefly discuss our results.

\section{Classical Method}

The classical method for finding symmetry reductions of partial differential equations is the Lie group method of infinitesimal transformations (cf., Olver 1993). Though this method is entirely algorithmic, it often involves a large amount of tedious algebra and auxiliary calculations which can become virtually unmanageable if attempted manually, and so symbolic manipulation programs have been developed, for example in MACSYMA, MAPLE, MATHEMATICA, and REDUCE, to facilitate the calculations. An excellent survey of the different packages presently available and a discussion of their strengths and applications is given by Hereman (1994). In this paper we the associated system of determining equations was generated using the MACSYMA program symmgrp.max (Champagne et al. 1991). Subsequently this system was analysed interactively using the MAPLE package diffgrob2, and the Reid strategy for controlling expression swell was employed (cf. Clarkson \& Mansfield 1994c; Mansfield 1993).

To apply the classical method to the system (1.6) we consider the one-parameter Lie group of infinitesimal transformations in $(x, y, t, u, v)$ given by

$$
\begin{aligned}
\tilde{x} & =x+\varepsilon \xi_{1}(x, y, t, u, v)+O\left(\varepsilon^{2}\right), \\
\tilde{y} & =y+\varepsilon \xi_{2}(x, y, t, u, v)+O\left(\varepsilon^{2}\right), \\
\tilde{t} & =t+\varepsilon \xi_{3}(x, y, t, u, v)+O\left(\varepsilon^{2}\right), \\
\tilde{u} & =u+\varepsilon \phi_{1}(x, y, t, u, v)+O\left(\varepsilon^{2}\right), \\
\tilde{v} & =v+\varepsilon \phi_{2}(x, y, t, u, v)+O\left(\varepsilon^{2}\right),
\end{aligned}
$$

where $\varepsilon$ is the group parameter. Then one requires that this transformation leaves invariant the set

$$
\mathcal{S}_{\boldsymbol{\Delta}} \equiv\left\{u(x, y, t), v(x, y, t): \Delta_{1}(u, v)=0, \Delta_{2}(u, v)=0\right\},
$$

of solutions of the system (1.6). This yields an overdetermined, linear system of equations for the infinitesimals $\xi_{i}(x, y, t, u, v), i=1,2,3$, and $\phi_{j}(x, y, t, u, v), j=1,2$. The associated Lie algebra of infinitesimal symmetries is the set of vector fields of the form

$$
\mathbf{v}=\xi_{1} \partial_{x}+\xi_{2} \partial_{y}+\xi_{3} \partial_{t}+\phi_{1} \partial_{u}+\phi_{2} \partial_{v}
$$

where $\partial_{x} \equiv \partial / \partial x$ etc. Having determined the infinitesimals, the symmetry variables are found by solving the invariant surface conditions

$$
\begin{aligned}
& \psi_{1} \equiv \xi_{1} u_{x}+\xi_{2} u_{y}+\xi_{3} u_{t}-\phi_{1}=0, \\
& \psi_{2} \equiv \xi_{1} v_{x}+\xi_{2} v_{y}+\xi_{3} v_{t}-\phi_{2}=0 .
\end{aligned}
$$

Applying the classical method to the system (1.6) yields a system of twenty-one one-term equations which have the solution

$$
\xi_{1}=f_{1}(x), \quad \xi_{2}=f_{2}(y), \quad \xi_{3}=f_{3}(t), \quad \phi_{1}=f_{4}(t), \quad \phi_{2}=f_{5}(x)+f_{6}(y)+f_{7}(t)
$$


where the $f_{i}$ are arbitrary functions of their arguments. The interpretation of this symmetry group is, that if $u=U(x, y, t), v=V(x, y, t)$ is a solution of (1.6) then another solution is $u(x, y, t)=U\left(f_{1}(x), f_{2}(y), f_{3}(t)\right)+f_{4}(t)$ and $v(x, y, t)=V\left(f_{1}(x), f_{2}(y), f_{3}(t)\right)+f_{5}(x)+f_{6}(y)+f_{7}(t)$. We shall use this classical symmetry group to simplify the presentation of the nonclassical reduction solutions obtained below.

There are two canonical, classical, symmetry reductions.

$\underline{\text { Reduction } 2.1} \xi_{1}=-\mu, \xi_{2}=1, \xi_{3}=\phi_{1}=\phi_{2}=0$. In this case we obtain the symmetry reduction

$$
u(x, y, t)=P(z, t), \quad v(x, y, t)=Q(z, t), \quad z=x+\mu y,
$$

where $P(z, t)$ and $Q(z, t)$ satisfy

$$
P_{z z t}+2 P_{z} Q_{z t}=0, \quad Q_{z z}=P_{z}^{2}
$$

This system can be viewed as a form of the real, unpumped Maxwell-Bloch system; see equation (4.1) below with $c=0$, which arises in nonlinear optics (cf., Ablowitz \& Segur 1981). Further, making the transformation $P=\frac{1}{2} \theta, Q_{z z}=\frac{1}{4} \theta_{z}^{2}$ and $Q_{z t}=-\frac{1}{2} \cos \theta$ in (2.6) yields the sineGordon equation (1.2). We remark that since the system (1.6) is invariant under the transformation $(x, y) \rightarrow(f(x), g(y))$, where $f(x)$ and $g(y)$ are arbitrary functions, then we can generate a variety of other, equivalent, reductions.

Reduction $2.2 \xi_{1}=\kappa_{1}, \xi_{2}=\kappa_{2}, \xi_{3}=1, \phi_{1}=\phi_{2}=0$. In this case we obtain the symmetry reduction

$$
u(x, y, t)=P(z, \zeta), \quad v(x, y, t)=Q(z, \zeta), \quad z=x-\kappa_{1} t, \quad \zeta=y-\kappa_{2} t,
$$

where $P(z, \zeta)$ and $Q(z, \zeta)$ satisfy

$$
\begin{aligned}
& \kappa_{1} P_{z z \zeta}+\kappa_{2} P_{z \zeta \zeta}+P_{z} P_{\zeta}\left(\kappa_{1} P_{z}+\kappa_{2} P_{\zeta}\right)+\kappa_{1} P_{\zeta} Q_{z z}+\kappa_{2} P_{z} Q_{\zeta \zeta}=0 \\
& Q_{z \zeta}=P_{z} P_{\zeta}
\end{aligned}
$$

\section{Nonclassical Method}

Bluman \& Cole (1969), in their study of symmetry reductions of the linear heat equation, proposed the so-called "nonclassical method of group-invariant solutions"; this technique is also known as the "method of conditional symmetries" (cf., Levi \& Winternitz 1989). This method involves considerably more algebra and associated calculations than the classical Lie method. In fact, it has been suggested that for some partial differential equations, the calculation of these nonclassical reductions might be too difficult to do explicitly, especially if attempted manually, since the associated determining equations are now an overdetermined, nonlinear system. Further, the associated vector fields arising from the nonclassical method do not form a vector space, still less a Lie algebra, since the invariant surface conditions (2.4) depend upon the particular reduction.

In the nonclassical method one requires only the subset of $\mathcal{S}_{\boldsymbol{\Delta}}$ given by

$$
\mathcal{S}_{\boldsymbol{\Delta}, \boldsymbol{\psi}}=\left\{u(x, y, t), v(x, y, t): \Delta_{1}(u, v)=0, \Delta_{2}(u, v)=0, \psi_{1}(u, v)=0, \psi_{2}(u, v)=0\right\},
$$

is invariant under the transformation (2.1), where $\mathcal{S}_{\boldsymbol{\Delta}}$ is as defined in $(2.2)$ and $\psi_{1}=0$ and $\psi_{2}=0$ are the invariant surface conditions (2.4).

As we showed in Clarkson \& Mansfield (1994c), the standard procedure for applying the nonclassical method (e.g., as described by Levi \& Winternitz 1989), can create difficulties, particularly when implemented in symbolic manipulation programs. These difficulties often arise 
for equations such as (1.6) which require the use of differential consequences of the invariant surface conditions (2.4). In Clarkson \& Mansfield (1994c), we proposed an algorithm for calculating the determining equations associated with the nonclassical method which avoids these difficulties, and we use that algorithm here. Further we showed how the MACSYMA package symmgrp.max, which was written to calculate the determining equations for the classical method, can be adapted to calculate the determining equations for the nonclassical method and we use this modification here.

To apply the nonclassical method to the system (1.6), there are three cases to consider: (i), $\xi_{3} \neq 0,(\mathrm{ii}), \xi_{3}=0$ and $\xi_{2} \neq 0$, and (iii), $\xi_{3}=0$ and $\xi_{2}=0$ and $\xi_{1} \neq 0$.

\subsection{Case (i). $\xi_{3} \neq 0$}

In this case we set $\xi_{3}=1$, without loss of generality, and so the invariant surface conditions (2.4) simplify to

$$
\xi_{1} u_{x}+\xi_{2} u_{y}+u_{t}=\phi_{1}(x, y, t, u, v), \quad \xi_{1} v_{x}+\xi_{2} v_{y}+v_{t}=\phi_{2}(x, y, t, u, v) .
$$

We first eliminate $u_{x y t}, v_{x t}$ and $v_{y t}$ from (1.6) using (3.2) and then apply the classical Lie algorithm to the resulting equations. This procedure yields a system of twenty-eight determining equations, of which twenty were linear and eight nonlinear. Then using the MAPLE package diffgrob2 (Mansfield 1993) these simplify to seventeen equations, thirteen of which are one-term equations and the other four are two-term nonlinear equations. This reduced system is easily solved to yield the classical infinitesimals (2.5). Thus, in this generic case, the nonclassical method does not yield any additional symmetry reductions to those obtained using the classical Lie method.

\subsection{Case (ii). $\xi_{3}=0, \xi_{2} \neq 0$}

In this case we set $\xi_{2}=1$, without loss of generality, and, analogous to the procedure in the generic case, we use (2.4), with $\xi_{3}=0$ and $\xi_{2}=1$, to eliminate all $y$-derivatives, i.e. $u_{x y t}, u_{y}, v_{y t}$ and $v_{x y}$, from (1.6). Then applying the classical Lie algorithm to the resulting equations yields the following determining equations,

$$
\begin{aligned}
& \xi_{1, v}=0, \quad \xi_{1, u}=0, \quad \xi_{1, t}=0, \quad \xi_{1, x} \xi_{1, y}-\xi_{1} \xi_{1, x y}=0, \\
& \phi_{1, u}=0, \quad \phi_{1, v}=0, \quad \phi_{2, u}=0, \quad \phi_{2, v}=0, \\
& \xi_{1} \phi_{1, y}-\xi_{1}^{2} \phi_{1, x}-\xi_{1, y} \phi_{1}=0, \\
& \xi_{1} \phi_{2, y t}-\xi_{1}^{2} \phi_{2, x t}-\xi_{1, y} \phi_{2, t}=0, \\
& \xi_{1} \phi_{2, x y}-\xi_{1, y} \phi_{2, x}-\xi_{1} \phi_{1} \phi_{1, x}=0, \\
& \xi_{1} \phi_{1} \phi_{2, x t}+\xi_{1} \phi_{1, x} \phi_{2, t}+\xi_{1} \phi_{1, x y t}-\xi_{1, y} \phi_{1, x t}=0 \text {. }
\end{aligned}
$$

The system (3.3) is straightforward to analyse. From $(3.3 a, b, c, d)$, one obtains that,

$$
\xi_{1}=g_{2}^{\prime}(y) / g_{1}^{\prime}(x), \quad \phi_{1}=g_{2}^{\prime}(y) w_{1}(z, t), \quad \phi_{2}=g_{2}^{\prime}(y)\left[w_{2}(z, t)+w_{3}(x, y)\right]
$$

where $z=g_{1}(x)+g_{2}(y)$, with $g_{1}(x)$ and $g_{2}(y)$ arbitrary functions. Substituting these into (3.3e,f) it is obtained that,

$$
\begin{aligned}
w_{1} w_{2, t}+w_{1, z t}+m_{1}(t) & =0, \\
2 w_{2, z}-w_{1}^{2}+m_{2}(t) & =0, \\
w_{3}+H(x)+K(y) & =0,
\end{aligned}
$$

where $m_{1}(t), m_{2}(t), H(x)$ and $K(y)$ are arbitrary functions of integration (we have set a third arbitrary function of $z$ to zero, without loss of generality). It transpires that after the solutions $u$ and $v$ have been obtained, we can use the classical symmetries $(2.5)$ to set $g_{1}(x)=x, g_{2}(y)=y$, 
$H(x)=0$ and $K(y)=0$. Thus, we do this now, to keep the exposition simple. Putting everything together, we obtain the following solution to the determining equations,

$$
\xi_{1}=1, \quad \xi_{2}=1, \quad \xi_{3}=0, \quad \phi_{1}=w_{1}(z, t), \quad \phi_{2}=w_{2}(z, t),
$$

where $z=x+y$ and $w_{1}(z, t)$ and $w_{2}(z, t)$ satisfy $(3.4 a, b)$.

We digress to remark that eliminating $w_{2}$ from $(3.4 a, b)$ and setting $w_{1}=w$ yields,

$$
w w_{z z t}-w_{z} w_{z t}-m_{1}(t) w_{z}+w^{3} w_{t}-\frac{1}{2} \frac{\mathrm{d} m_{2}}{\mathrm{~d} t} w^{2}=0
$$

In $\S 6.2$ below we apply the Painlevé test due to Weiss et al. (1993) and discuss the integrability of this equation. We note that in the special case when $m_{1}(t) \propto \frac{\mathrm{d} m_{2}}{\mathrm{~d} t}$, we can simplify this equation by rescaling $t$. In this case, it is sufficient to set $m_{1}(t)=\mu_{1}$ and $m_{2}(t)=\mu_{2} t$, with $\mu_{1}$ and $\mu_{2}$ constants, without loss of generality and so we obtain,

$$
w w_{z z t}-w_{z} w_{z t}-\mu_{1} w_{z}+w^{3} w_{t}-\frac{1}{2} \mu_{2} w^{2}=0 .
$$

Next, we set $w_{1}(z, t)=2 P_{z}(z, t)$ and $w_{2}(z, t)=2 Q_{z}(z, t)$ in (3.5) and then integrate the invariant surface conditions (2.4) using the method of characteristics. Hence we obtain the nonclassical reduction of (1.6) given by

$$
u(x, y, t)=P(z, t)+\tilde{P}(\tilde{z}, t), \quad v(x, y, t)=Q(z, t)+\tilde{Q}(\tilde{z}, t)
$$

where $z=x+y$ and $\tilde{z}=x-y, P(z, t)$ and $Q(z, t)$ satisfy

$$
\begin{aligned}
P_{z z t}+2 P_{z} Q_{z t}+\frac{1}{2} m_{1}(t) & =0, \\
Q_{z z}-P_{z}^{2}+\frac{1}{4} m_{2}(t) & =0,
\end{aligned}
$$

$\tilde{P}(\tilde{z}, t)$ and $\tilde{Q}(\tilde{z}, t)$ satisfy the same equations, and $m_{1}(t)$ and $m_{2}(t)$ are arbitrary functions, which are effectively separation functions.

In $\S 4$ below, we obtain exact solutions of the sine-Gordon system (1.6), by analysing the reduced system (3.9), or equivalently (3.6), for some special choices of $m_{1}(t)$ and $m_{2}(t)$.

$$
\text { 3.3. Case (iii). } \xi_{3}=0, \xi_{2}=0, \xi_{1} \neq 0
$$

In this case we set $\xi_{1}=1$, without loss of generality, and, as above, we use (2.4), with $\xi_{3}=0$, $\xi_{2}=0$ and $\xi_{1}$, to eliminate all $x$-derivatives, i.e. $u_{x y t}, u_{x}, v_{x t}$ and $v_{x y}$, from (1.6). Then applying the classical Lie algorithm to the resulting equations yields seven equations containing over twelve hundred terms. Since this system is considerably more complex than the sine-Gordon system (1.6) we are studying, we shall not pursue this case further here.

\subsection{Nonclassical reductions of (1.1) and (1.3)}

In this subsection, we record, for the sake of completeness, some nonclassical reductions of the $2+1$-dimensional sine-Gordon system (1.1) and the $2+1$-dimensional sine-Gordon equation (1.3). By considering the gauge transformation (1.5) and the nonclassical reduction (3.8) of (1.6) in the case when $m_{1} \equiv 0$ and $m_{2} \equiv 0$, it is straightforward to obtain the nonclassical reduction of the $2+1$-dimensional sine-Gordon system (1.1) given by

$$
\Theta(X, Y, T)=\theta(X, T)+\tilde{\theta}(Y, T), \quad \Phi(X, Y, T)=\phi(X, T)+\tilde{\phi}(Y, T),
$$

where $\theta(X, T), \tilde{\theta}(Y, T), \phi(X, T)$ and $\tilde{\phi}(Y, T)$ satisfy

$$
\begin{array}{ll}
\theta_{X T}=F(T) \sin \theta, & \phi_{X T}=-\tilde{F}(T) \sin \phi \\
\tilde{\theta}_{Y T}=\tilde{F}(T) \sin \tilde{\theta}, & \tilde{\phi}_{Y T}=-F(T) \sin \tilde{\phi},
\end{array}
$$


where $F(T)$ and $\tilde{F}(T)$ are arbitrary functions.

The analogous nonclassical reduction of the 2+1-dimensional sine-Gordon equation (1.3) given by

$$
\Theta(X, Y, T)=\theta(X, T)+\tilde{\theta}(Y, T)
$$

where $\theta(X, T)$ and $\tilde{\theta}(Y, T)$ satisfy

$$
\theta_{X T}=F(T) \sin \theta, \quad \tilde{\theta}_{Y T}=-F(T) \sin \tilde{\theta},
$$

where $F(T)$ is an arbitrary function.

\section{Some Exact Solutions of the sine-Gordon system (1.6)}

\subsection{Nonclassical reduction solutions and the Maxwell-Bloch system}

Equations (3.8) describe nonclassical reductions to the $2+1$-dimensional sine-Gordon system (1.6), in terms of the system $(3.9)$, or equivalently $(3.4 a, b)$. System $(3.4 a, b)$ is easily seen to be a generalisation of the real, pumped, Maxwell-Bloch system,

$$
E_{\chi}=\rho, \quad \rho_{\tau}=E \eta, \quad \eta_{\tau}+E \rho=c,
$$

where $c$ is a constant, which has a physical interpretation in nonlinear optics (Burtsev \& Gabitov 1994). Eliminating $\rho$, and setting $\chi \equiv t, \tau \equiv z, E \equiv w_{1}$ and $\eta \equiv-w_{2, t}$ in (4.1) yields $(3.4 a, b)$ with $m_{1} \equiv 0$ and $m_{2} \equiv 2 c t$. For $c=0$, the unpumped case, the system (4.1) is equivalent to the sine-Gordon equation (1.2), which is solvable by inverse scattering (Ablowitz et al. 1974). However for $c \neq 0$, the system (4.1) is solvable by inverse scattering with a non-isospectral Lax pair (Burtsev et al. 1987); see $\S 6.2$ below. Several authors have obtained classical reductions of (4.1) to the fifth Painlevé equation (see, for example Burtsev 1993; Kitaev et al. 1993; Schief 1994; Winternitz 1992), and a Bäcklund transformation for (4.1) is discussed by Schief (1994).

In this section, we examine some special cases of the system (3.9), or equivalently (3.6). First, in the case $m_{1}(t)=m_{2}(t) \equiv 0$, we express solutions of the system in terms of solutions of the sineGordon equation (1.2). This yields solutions of (1.6) that can be written as a decoupled sum of solutions of the sine-Gordon equation, with different scalings of the time variable in each summand being allowed. Second, in the case $m_{1}(t) \propto \frac{\mathrm{d} m_{2}}{\mathrm{~d} t}(t)$, we obtain scaling reductions and travelling wave solutions which can be expressed in terms of solutions of the second, third, and fifth Painlevé equations. For each of these cases, we give the necessary transformations to obtain the associated solutions of (1.6), and write down some examples. Indeed, hierarchies of these solutions of (1.6) are obtained, with explicit transformations connecting the members of each hierarchy given.

$$
\text { 4.2. The case } m_{1}(t)=m_{2}(t) \equiv 0
$$

First we note that in this case, the system (3.4) has a classical symmetry which allows an arbitrary rescaling of the $t$ variable. As noted in $\S 2$, the transformation $P=\frac{1}{2} \theta, Q_{z z}=\frac{1}{4} \theta_{z}^{2}$ and $Q_{z t}=-\frac{1}{2} \cos \theta$ maps (3.9) with $m_{1}(t)=m_{2}(t)=0$ into the sine-Gordon equation (1.2). Hence in this case we obtain the solution to (1.6) given by

$$
\begin{aligned}
& u(x, y, t)=\frac{1}{2} \theta(x+y, \tau(t))+\frac{1}{2} \tilde{\theta}(x-y, \tilde{\tau}(t)) \\
& v(x, y, t)=\frac{1}{4} \int^{x+y} \int^{z} \theta(\zeta, \tau(t)) \mathrm{d} \zeta \mathrm{d} z+\frac{1}{4} \int^{x-y} \int^{\tilde{z}} \tilde{\theta}(\tilde{\zeta}, \tilde{\tau}(t)) \mathrm{d} \tilde{\zeta} \mathrm{d} \tilde{z},
\end{aligned}
$$

where $\theta(z, \tau)$ and $\tilde{\theta}(\tilde{z}, \tilde{\tau})$ are any two solutions of (1.2). Three well-known solutions of (1.2) are

$$
\begin{aligned}
& \theta(z, t)=4 \tan ^{-1}\{C \exp (\beta z+t / \beta)\}, \\
& \theta(z, t)=4 \tan ^{-1}\left\{\frac{\lambda}{\mu} \sin \left(\mu z-\frac{\mu t}{\lambda^{2}+\mu^{2}}\right) \operatorname{sech}\left(\lambda z+\frac{\lambda t}{\lambda^{2}+\mu^{2}}\right)\right\}, \\
& \theta(z, t)=4 \tan ^{-1}\left\{\left(\frac{\beta_{2}+\beta_{1}}{\beta_{2}-\beta_{1}}\right) \frac{\exp \left(\eta_{2}\right)-\exp \left(\eta_{1}\right)}{1+\exp \left(\eta_{1}+\eta_{2}\right)}\right\},
\end{aligned}
$$


where $\eta_{1}=\beta_{1} z+t / \beta_{1}$, and $\eta_{2}=\beta_{2} z+t / \beta_{2}$. The first of these is the one-soliton, or kink, solution, the second (4.4), is the "breather" solution, while the third, (4.5), is the kink-kink solution (if $\beta_{2}>-\beta_{1}>0$ ) or kink-antikink solution (if $\beta_{2}>\beta_{1}>0$ ). In Figure 1 we plot $(4.2 a)$ when $\theta(z, \tau)$ and $\tilde{\theta}(\tilde{z}, \tilde{\tau})$ are both breathers and in Figure 2 when $\theta(z, \tau)$ and $\tilde{\theta}(\tilde{z}, \tilde{\tau})$ are both kink-kink solutions. Since one can rescale $x, y$ and $t$ arbitrarily, using the classical symmetries of (1.6), a wide variety of solutions is obtainable. This is illustrated in Figure 3 where $\theta(z, \tau)$ and $\tilde{\theta}(\tilde{z}, \tilde{\tau})$ are both breather solutions but $x$ has been scaled to $-\exp \left(1-x^{2}\right), \tau(t)=\exp \left(1-t^{2}\right)$ and $\tilde{\tau}(t)=-\exp \left(1-t^{2}\right)$. This last figure illustrates the fundamental difficulties of attempting to solve the system (1.6) numerically. In particular, one would not be able to distinguish the solutions in an initial value problem since an exponentially small change in the initial conditions can result in completely different qualitative behaviours.

$$
\text { 4.3. The case } m_{1}(t) \propto \frac{\mathrm{d} m_{2}}{\mathrm{~d} t}(t)
$$

To obtain exact solutions in this case, it is sufficient to analyse (3.7), which has a threedimensional group of classical Lie point symmetries, given by the vector fields

$$
\mathbf{v}_{1}=\partial_{z}, \quad \mathbf{v}_{2}=\partial_{t}, \quad \mathbf{v}_{3}=2 t \partial_{t}-z \partial_{z}+w \partial_{w}
$$

The first two vector fields yield the travelling wave reduction

$$
w(z, t)=\eta(\zeta), \quad \zeta=z-\beta t
$$

where $\eta(\zeta)$ satisfies

$$
\eta_{\zeta \zeta}=-\frac{1}{2} \eta^{3}-\mu_{2} \frac{\zeta+\kappa_{1}}{2 \beta} \eta+\frac{\mu_{1}}{\beta},
$$

where $\kappa_{1}$ is a constant of integration. Equation (4.7) is equivalent to second Painlevé equation $\left(\mathrm{P}_{I I}\right)$

$$
y_{x x}=2 y^{3}+x y+\alpha
$$

(cf., Ince 1956), provided that $\mu_{2} \neq 0$; if $\mu_{2}=0$ then (4.7) is solvable in terms of Jacobi elliptic functions (cf., Whittaker \& Watson 1927).

The third vector field $\mathbf{v}_{3}$ yields the scaling reduction

$$
w(z, t)=z^{-1} F(\zeta), \quad \zeta=z t^{1 / 2},
$$

where $F(\zeta)$ satisfies

$$
\zeta^{2} F F_{\zeta \zeta \zeta}-\zeta^{2} F_{\zeta} F_{\zeta \zeta}+\zeta F F_{\zeta \zeta}+F^{3} F_{\zeta}+2 \mu_{1}\left(\zeta F-\zeta^{2} F_{\zeta}\right)-\mu_{2} \zeta F^{2}=0 .
$$

In the case $\mu_{1}=0$, this is exactly equation (5.5) of Winternitz (1992), with $c=\frac{1}{8}$. Equation (4.9) has a first integral of the form,

$$
\left(F_{\zeta \zeta}-\mu_{2} F+2 \mu_{1}\right)^{2} \zeta^{2}+F^{2}\left\{\left(F_{\zeta}\right)^{2}-\mu_{2} F^{2}+4 \mu_{1} F-K\right\}=0 .
$$

We note here that this integral appears not to be obtainable by either the classical or contact symmetry methods. Following the analysis in Bureau (1972), p. 212, we can write the solutions of (4.10) in terms of the third and fifth Painlevé equations. It transpires that there are three cases to consider, (i) $\mu_{1}$ arbitrary and $\mu_{2} \neq 0$, (ii) $\mu_{1} \neq 0$ and $\mu_{2}=0$, and (iii) $\mu_{1}=\mu_{2}=0$.

Case 4.3(i). $\mu_{1}$ arbitrary and $\mu_{2} \neq 0$. In this case, the transformation to be used in (4.10), as given by Bureau's method, is

$$
F(\zeta)=\frac{\mathrm{i}}{\psi(\xi)[\psi(\xi)-1]}\left\{2 \xi \psi_{\xi}(\xi)+1-\psi(\xi)-\frac{2 \mathrm{i} \mu_{1}}{\mu_{2}}[\psi(\xi)-1]^{2}\right\}, \quad \xi=\frac{1}{2} \zeta^{2},
$$


where $\psi(\xi)$ satisfies the fifth Painlevé equation $\left(\mathrm{P}_{V}\right)$,

$$
\psi_{\xi \xi}=\left(\frac{1}{2 \psi}+\frac{1}{\psi-1}\right) \psi_{\xi}^{2}-\frac{1}{\xi} \psi_{\xi}+\frac{(\psi-1)^{2}}{\xi^{2}}\left(\alpha_{5} \psi+\frac{\beta_{5}}{\psi}\right)+\frac{\gamma_{5}}{\xi} \psi+\frac{\delta_{5} \psi(\psi+1)}{\psi-1},
$$

and the constants are given by

$$
\alpha_{5}=\left(K \mu_{2}-4 \mu_{1}^{2}\right) /\left(8 \mu_{2}^{2}\right), \quad \beta_{5}=-\left(\mu_{2}-2 \mathrm{i} \mu_{1}\right)^{2} /\left(8 \mu_{2}^{2}\right), \quad \gamma_{5}=-\frac{1}{4} \mu_{2}, \quad \delta_{5}=0 .
$$

It is known that the special case of $\mathrm{P}_{V}$ with $\delta_{5}=0$ can always be solved in terms of solutions of the third Painlevé equation $\left(\mathrm{P}_{I I I}\right)$

$$
\phi_{\zeta \zeta}=\frac{1}{\phi}\left(\phi_{\zeta}\right)^{2}-\frac{1}{\zeta} \phi_{\zeta}+\frac{\alpha_{3} \phi^{2}+\beta_{3}}{\zeta}+\gamma_{3} \phi^{3}+\frac{\delta_{3}}{\phi}
$$

(cf., Fokas \& Ablowitz 1982; Gromak 1975); Cosgrove \& Scoufis (1993), remark that there are infinitely many other special cases of $\mathrm{P}_{V}$ that are solvable in terms of solutions of $\mathrm{P}_{I I I}$. Combining (4.11) with the transformation of $\mathrm{P}_{V}$ to $\mathrm{P}_{I I I}$ given in Gromak (1975), one obtains the transformation to be used in (4.10) to be

$$
F(\zeta)=-\mathrm{i} \zeta\left\{\phi_{\zeta}(\zeta)+c \phi^{2}(\zeta)+d\right\} / \phi(\zeta)
$$

where $\phi(\zeta)$ satisfies $\mathrm{P}_{I I I}$ (4.13) with constants given by

$$
\alpha_{3}=\frac{\sqrt{K \mu_{2}-4 \mu_{1}^{2}}+\mu_{2}-2 \mathrm{i} \mu_{1}}{4 d}, \quad \beta_{3}=\frac{\sqrt{K \mu_{2}-4 \mu_{1}^{2}}+\mu_{2}+2 \mathrm{i} \mu_{1}}{4 c}, \quad \gamma_{3}=c^{2}, \quad \delta_{3}=-d^{2},
$$

with $\mu_{2}=-4 c d$.

Case 4.3(ii). $\mu_{1} \neq 0$ and $\mu_{2}=0$. In this case, the transformation used is

$$
F(\zeta)=\mathrm{i} \zeta\left[\phi_{\zeta}(\zeta)-\frac{1}{2} \nu\right] / \phi(\zeta)
$$

where $\nu$ is a non-zero constant and $\phi(\zeta)$ satisfies $\mathrm{P}_{I I I}$ (4.13) with constants $\alpha_{3}=2 \mathrm{i} \mu_{1} / \nu$, $\beta_{3}=\frac{1}{8} \nu\left(4+\mathrm{i} K / \mu_{1}\right), \gamma_{3}=0$ and $\delta_{3}=-\frac{1}{4} \nu^{2}$.

Case 4.3(iii). $\mu_{1}=\mu_{2}=0$. In this case, the transformation used is $F(\zeta)=\mathrm{i} \zeta \phi_{\zeta}(\zeta) / \phi(\zeta)$, where $\phi(\zeta)$ satisfies $\mathrm{P}_{I I I}$ (4.13) with constants $\alpha_{3}=1, \beta_{3}=-\frac{1}{4} K$ and $\gamma_{3}=\delta_{3}=0$.

In the following two subsections, we write down the solutions to (1.6) in terms of solutions of $\mathrm{P}_{I I}$ and $\mathrm{P}_{I I I}$. Using some known rational and other solutions of these Painlevé equations, we obtain some simple explicit solutions for the sine-Gordon system (1.6). Further, we obtain Bäcklund transformations for each subfamily of solutions.

\subsection{Nonclassical solutions in terms of the second Painlevé equation}

Here we consider travelling wave reductions to (3.7) given by (4.6), to obtain solutions to (1.6) in terms of $\mathrm{P}_{I I}$ (4.8). We set $\mu_{2}=1$ in (4.7) for convenience. Following the method of integration in $\S 3.2$, we obtain the solution to (1.6), after a complex rescaling of $\eta(\zeta)$ and simplifying using the classical symmetry group,

$$
\begin{aligned}
u(x, y, t)=\mathrm{i}\{ & \left.\int^{\zeta} \eta\left(\zeta_{1} ; \mu_{1}, 1, \beta\right) \mathrm{d} \zeta_{1}+\int^{\tilde{\zeta}} \tilde{\eta}\left(\tilde{\zeta}_{1} ; \mu_{1}, 1, \tilde{\beta}\right) \mathrm{d} \tilde{\zeta}_{1}\right\} \\
v(x, y, t)=- & \int^{\zeta} \int^{\zeta_{1}}\left[\eta\left(\zeta_{2} ; \mu_{1}, 1, \beta\right)\right]^{2} \mathrm{~d} \zeta_{2} \mathrm{~d} \zeta_{1}-\int^{\tilde{\zeta}} \int^{\tilde{\zeta}_{1}}\left[\tilde{\eta}\left(\tilde{\zeta}_{2} ; \mu_{1}, 1, \tilde{\beta}\right)\right]^{2} \mathrm{~d} \tilde{\zeta}_{2} \mathrm{~d} \tilde{\zeta}_{1} \\
& -\frac{1}{4}\left(x^{2}+y^{2}\right) t+\frac{1}{8}[\beta(x+y)+\tilde{\beta}(x-y)] t^{2}-\frac{1}{4}\left[\kappa_{1}(x+y)+\kappa_{2}(x-y)\right] t,
\end{aligned}
$$


where $\eta\left(\zeta ; \mu_{1}, \mu_{2}, \beta\right)$ and $\tilde{\eta}\left(\tilde{\zeta} ; \mu_{1}, \mu_{2}, \tilde{\beta}\right)$ satisfy (4.7). It is well-known that $\mathrm{P}_{I I}$ (4.8) possesses rational solutions and one-parameter families of solutions expressible in terms of Airy functions for special choices of the parameter $\alpha$ (cf., Airault 1979; Clarkson 1990; Gibbon et al. 1988; Murato, 1985). For example, the first three non-zero rational solutions $y(x ; \alpha)$ of $\mathrm{P}_{I I}(4.8)$ are given by

$$
y(x ; 1)=-\frac{1}{x}, \quad y(x ; 2)=-\frac{2\left(x^{3}-2\right)}{x\left(x^{3}+4\right)}, \quad y(x ; 3)=-\frac{3 x^{2}\left(x^{6}+8 x^{3}+160\right)}{\left(x^{3}+4\right)\left(x^{6}+20 x^{3}-80\right)} .
$$

Thus, we obtain the simple solution of (1.6),

$$
\begin{aligned}
& u_{1}(x, y, t)=\mathrm{i} \ln (\zeta \tilde{\zeta}), \\
& v_{1}(x, y, t)=\ln (\zeta \tilde{\zeta})-\frac{1}{4}\left(x^{2}+y^{2}\right) t-\frac{1}{8}[c(x+y)+\beta(x-y)] t^{2},
\end{aligned}
$$

while setting $c=\beta=-\frac{1}{2}$, and then using the classical symmetry to rescale $t$ (we let $t$ go to $2 t$ for convenience) so that $\zeta=x+y+t$ and $\tilde{\zeta}=x-y+t$, we obtain,

$$
\begin{aligned}
& u_{2}(x, y, t)=\mathrm{i}\left\{\ln (\zeta \tilde{\zeta})-\ln \left[\left(\zeta^{3}+4\right)\left(\tilde{\zeta}^{3}+4\right)\right]\right\} \\
& v_{2}(x, y, t)=\ln \left[\zeta \tilde{\zeta}\left(\zeta^{3}+4\right)\left(\tilde{\zeta}^{3}+4\right)\right]-\frac{1}{4}\left(x^{2}+y^{2}\right) t-\frac{1}{2} x t^{2} \\
& u_{3}(x, y, t)=\mathrm{i}\left\{\ln \left[\left(\zeta^{3}+4\right)\left(\tilde{\zeta}^{3}+4\right)\right]-\ln \left[\left(\zeta^{6}+20 \zeta^{3}-80\right)\left(\tilde{\zeta}^{6}+20 \tilde{\zeta}^{3}-80\right)\right]\right\} \\
& v_{3}(x, y, t)=\ln \left[\left(\zeta^{3}+4\right)\left(\tilde{\zeta}^{3}+4\right)\left(\zeta^{6}+20 \zeta^{3}-80\right)\left(\tilde{\zeta}^{6}+20 \tilde{\zeta}^{3}-80\right)\right]-\frac{1}{2}\left(x^{2}+y^{2}\right) t-\frac{1}{2} x t^{2}
\end{aligned}
$$

It can be seen that these solutions are not obtainable from simple solutions using the Bäcklund transformation (5.2) for the system given in $\S 6$ below. However, one may use the well-known Bäcklund transformation for $\mathrm{P}_{I I}(4.8)$,

$$
y(x ; \alpha+1)=-y(x ; \alpha)-\frac{1+2 \alpha}{2 y^{2}(x ; \alpha)+2 y_{x}(x ; \alpha)+x}
$$

(cf., Airault 1979), to obtain transformations within the family of solutions (4.15). It is straightforward to show that

$$
\begin{gathered}
\int^{x} y\left(x_{1} ; \alpha+1\right) \mathrm{d} x_{1}=\int^{x} y\left(x_{1} ; \alpha\right) \mathrm{d} x_{1}-\ln \left\{2[y(x ; \alpha)]^{2}+2 y_{x}(x ; \alpha)+x\right\} \\
\int^{x} \int^{x_{1}}\left[y\left(x_{2} ; \alpha+1\right)\right]^{2} \mathrm{~d} x_{2} \mathrm{~d} x_{1}=-\int^{x} \int^{x_{1}}\left[y\left(x_{2} ; \alpha\right)\right]^{2} \mathrm{~d} x_{2} \mathrm{~d} x_{1}+\ln \left\{2[y(x ; \alpha)]^{2}+2 y_{x}(x ; \alpha)+x\right\} .
\end{gathered}
$$

Thus from (4.15),

$$
\begin{aligned}
\tilde{u}(x, y, t)=u(x, y, t) & -\mathrm{i} \ln \left[2 \eta^{2}\left(\zeta ; \mu_{1}, \mu_{2}, \beta\right)+2 \eta_{\zeta}\left(\zeta ; \mu_{1}, \mu_{2}, \beta\right)+\zeta\right] \\
& -\mathrm{i} \ln \left[2 \tilde{\eta}^{2}\left(\tilde{\zeta} ; \mu_{1}, \mu_{2}, \tilde{\beta}\right)+2 \tilde{\eta}_{\tilde{\zeta}}\left(\tilde{\zeta} ; \mu_{1}, \mu_{2}, \tilde{\beta}\right)+\tilde{\zeta}\right], \\
\tilde{v}(x, y, t)=v(x, y, t)+ & 2 \mathrm{i} u(x, y, t)+\ln \left[2 \eta^{2}\left(\zeta ; \mu_{1}, \mu_{2}, \beta\right)+2 \eta_{\zeta}\left(\zeta ; \mu_{1}, \mu_{2}, \beta\right)+\zeta\right] \\
+ & \ln \left[2 \tilde{\eta}^{2}\left(\tilde{\zeta} ; \mu_{1}, \mu_{2}, \tilde{\beta}\right)+2 \tilde{\eta}_{\tilde{\zeta}}\left(\tilde{\zeta} ; \mu_{1}, \mu_{2}, \tilde{\beta}\right)+\tilde{\zeta}\right]
\end{aligned}
$$

is the Bäcklund transformation acting within the family of solutions (4.15).

\subsection{Nonclassical solutions in terms of the third Painlevé equation}

The relationship between solutions of the ordinary differential equation (4.10) to solutions of the sine-Gordon system (1.6) is given by the following. If $F(\zeta)$ and $\hat{F}(\zeta)$ both satisfy (4.10), then 
the associated solution of (1.6) is given by,

$$
\begin{aligned}
& u(x, y, t)=\frac{1}{2} \int^{z t^{1 / 2}} \frac{F\left(\zeta_{1}\right)}{\zeta_{1}} \mathrm{~d} \zeta_{1}+\frac{1}{2} \int^{\hat{z} t^{1 / 2}} \frac{\hat{F}\left(\hat{\zeta}_{1}\right)}{\hat{\zeta}_{1}} \mathrm{~d} \hat{\zeta}_{1} \\
& v(x, y, t)=\frac{1}{4} \int^{z t^{1 / 2}} \int^{\zeta_{1}}\left[\frac{F\left(\zeta_{2}\right)}{\zeta_{2}}\right]^{2} \mathrm{~d} \zeta_{2} \mathrm{~d} \zeta_{1}+\frac{1}{4} \int^{\hat{z} t^{1 / 2}} \int^{\hat{\zeta}_{1}}\left[\frac{\hat{F}\left(\hat{\zeta}_{2}\right)}{\hat{\zeta}_{2}}\right]^{2} \mathrm{~d} \hat{\zeta}_{2} \mathrm{~d} \hat{\zeta}_{1}-\frac{1}{8} \mu_{2} t\left(z^{2}+\hat{z}^{2}\right),
\end{aligned}
$$

where $z=x+y$ and $\hat{z}=x-y$. In $\S 4.3$ above, we noted the relationship between the equation (4.10) and $\mathrm{P}_{I I I}$ (4.13). There are many known rational and one-parameter family solutions expressible in terms of Bessel functions for $\mathrm{P}_{I I I}$ (cf., Lukashevich 1965; Gromak 1977; Okamoto 1987), and Bäcklund transformations which map solutions of $\mathrm{P}_{I I I}$ into new solutions with different values of the parameters (cf., Airault 1979; Fokas \& Ablowitz 1982; Milne \& Clarkson 1993). In the following, we obtain examples of solutions for (1.6) associated with solutions of $\mathrm{P}_{I I I}$. Further, we discuss the relationship between the equation (4.10) and the third Painlevé equation (4.13), in more detail. The result will be hierarchies of solutions for the system (1.6), and transformations between solutions in each hierarchy.

Case 4.5(i) $\mu_{1}$ arbitrary, $\mu_{2} \neq 0$. The equations (4.10) and (4.13) are related by the system,

$$
\begin{aligned}
{\left[F_{\zeta}+2 \mathrm{i} \phi\left(\mathrm{i} c F+c+\alpha_{3}\right)\right]^{2}+F^{2} \mu_{2}-4 F \mu_{1}+K-F_{\zeta}^{2} } & =0 \\
\phi F+\mathrm{i} \zeta\left(\phi_{\zeta}+c \phi^{2}+d\right) & =0
\end{aligned}
$$

where $\mu_{1}=\mathrm{i}\left(\beta_{3} c-\alpha_{3} d\right), \mu_{2}=-4 c d$ and $K=-4\left(\alpha_{3}+c\right)\left(\beta_{3}+d\right)$, with $\gamma_{3}=c^{2}$ and $\delta_{3}=-d^{2}$. Hence we obtain the following result.

Theorem 4.5.1.

(i) If $F\left(\zeta ; \mu_{1}, \mu_{2}, K\right)$ is a solution of (4.10) then

$$
\phi\left(\zeta ; \alpha_{3}, \beta_{3}, \gamma_{3}, \delta_{3}\right)=\frac{\mathrm{i} \mu_{2}\left\{F_{\zeta}(\zeta) \mp\left[F_{\zeta}^{2}(\zeta)-\mu_{2} F^{2}(\zeta)+4 \mu_{1} F(\zeta)-K\right]^{1 / 2}\right\}}{2 \epsilon_{1}\left[\kappa+\mathrm{i} \mu_{2} F(\zeta)-2 \mathrm{i} \mu_{1}\right]},
$$

where $\kappa= \pm\left(\mu_{2} K-4 \mu_{1}^{2}\right)^{1 / 2}$, is a solution of the $P_{I I I}$ (4.13) with parameters

$$
\alpha_{3}=\epsilon_{1}\left(\kappa-\mu_{2}-2 \mathrm{i} \mu_{1}\right) / \mu_{2}, \quad \beta_{3}=-\left(\kappa-\mu_{2}+2 \mathrm{i} \mu_{1}\right) /\left(4 \epsilon_{1}\right), \quad \gamma_{3}=\epsilon_{1}^{2}, \quad \delta_{3}=-\mu_{2}^{2} /\left(16 \epsilon_{1}^{2}\right) .
$$

(ii) If $\phi\left(\zeta ; \alpha_{3}, \beta_{3}, \gamma_{3}, \delta_{3}\right)$ is a solution of $P_{I I I}$ (4.13) with $\gamma_{3}=\epsilon_{3}^{2}$ and $\delta_{3}=-\epsilon_{4}^{2}$, then

$$
F\left(\zeta ; \mu_{1}, \mu_{2}, K\right)=-\mathrm{i} \zeta\left[\phi_{\zeta}(\zeta)+\epsilon_{3} \phi^{2}(\zeta)+\epsilon_{4}\right] / \phi(\zeta)
$$

is a solution of (4.10) with parameters $\mu_{1}=\mathrm{i}\left(\beta_{3} \epsilon_{3}-\alpha_{3} \epsilon_{4}\right), \mu_{2}=-4 \epsilon_{3} \epsilon_{4}, K=-4\left(\alpha_{3}+\epsilon_{3}\right)\left(\beta_{3}+\epsilon_{4}\right)$.

Setting $\epsilon_{2}=-\mu_{2} /\left(4 \epsilon_{1}\right)$, and $\epsilon_{1}^{2}=\epsilon_{3}^{2}$ and $\epsilon_{2}^{2}=\epsilon_{4}^{2}$, then combining (4.22) and (4.21) yields the following Bäcklund transformation for the equation (4.10).

Theorem 4.5.2. If $F\left(\zeta ; \mu_{1}, \mu_{2}, K\right)$ is a solution of $(4.10)$, then

$\hat{F}\left(\zeta ; \hat{\mu}_{1}, \hat{\mu}_{2}, \hat{K}\right)=-\frac{\zeta}{\mu_{2} R}\left[2 \mathrm{i} \epsilon_{1} \mu_{2} R_{\zeta}\left(\kappa+\mathrm{i} \mu_{2} F-2 \mathrm{i} \mu_{1}\right)+4 \epsilon_{1}^{2} \epsilon_{4}\left(\kappa+\mathrm{i} \mu_{2} F-2 \mathrm{i} \mu_{1}\right)^{2}+2 \epsilon_{1} \mu_{2}^{2} F_{\zeta} R-\epsilon_{3} \mu_{2}^{2} R^{2}\right]$,

where

$$
R(\zeta)=F_{\zeta}(\zeta) \mp\left[F_{\zeta}^{2}(\zeta)-\mu_{2} F^{2}(\zeta)+4 \mu_{1} F(\zeta)-K\right]^{1 / 2}, \quad \kappa= \pm\left(\mu_{2} K-4 \mu_{1}^{2}\right)^{1 / 2},
$$


is also a solution of (4.10) with parameters

$$
\begin{aligned}
& \hat{\mu}_{1}=-\left[\mathrm{i}\left(\epsilon_{3} \mu_{2}+4 \epsilon_{1}^{2} \epsilon_{4}\right)\left(\kappa-\mu_{2}\right)+2\left(4 \epsilon_{1}^{2} \epsilon_{4}-\epsilon_{3} \mu_{2}\right) \mu_{1}\right] /\left(4 \epsilon_{1} \mu_{2}\right), \quad \hat{\mu}_{2}=-4 \epsilon_{3} \epsilon_{4}, \\
& \hat{K}=\frac{1}{\epsilon_{1} \mu_{2}}\left[\left(\epsilon_{3} \mu_{2}-2 \epsilon_{1} \mu_{2}-4 \epsilon_{1}^{2} \epsilon_{4}\right) \kappa+\left(\epsilon_{1}-\epsilon_{3}\right) \mu_{2}^{2}+\left(K-4 \epsilon_{3} \epsilon_{4}+4 \epsilon_{1} \epsilon_{4}\right) \epsilon_{1} \mu_{2}+2 \mathrm{i} \mu_{1}\left(\epsilon_{3} \mu_{2}+4 \epsilon_{1}^{2} \epsilon_{4}\right)\right] .
\end{aligned}
$$

As an example, we take the simple "seed solution" for $\mathrm{P}_{I I I}$ given by $\phi(\zeta ; a,-a, 1,1)=1$. Applying the transformation (4.22) to this solution yields the solution of (4.10) given by

$$
F\left(\zeta ;-2 \mathrm{i} a,-4,4\left(a^{2}-1\right)\right)=-2 \mathrm{i} \zeta .
$$

Applying the Bäcklund transformation (4.23) to (4.24) taking the upper sign and with $\epsilon_{1}=\epsilon_{2}=1$, $\epsilon_{3}=\epsilon_{4}=-1$ yields

$$
F\left(\zeta ; 2 \mathrm{i} a,-4,4\left(a^{2}-9\right)\right)=\frac{2 \mathrm{i} \zeta\left(4 \zeta^{2}+4 a \zeta+a^{2}+3\right)}{(2 \zeta+a-1)(2 \zeta+a+1)} .
$$

Alternatively, applying the Bäcklund transformation (4.23) to the solution (4.24) with $\epsilon_{1}=1$, $\epsilon_{2}=1, \epsilon_{3}=-1, \epsilon_{4}=1$ yields the solution to (4.10) given by

$$
F(\zeta ; 4 \mathrm{i},-4,4(a-3)(a+1))=4 \mathrm{i} \zeta /(2 \zeta+a-1) .
$$

It is known that $\mathrm{P}_{I I I}$ (4.13) possesses a one-parameter family of solutions characterised by the Riccati equation

$$
\phi_{\zeta}=-\gamma_{3}^{1 / 2} \phi^{2}+\left(1+\alpha_{3} \gamma_{3}^{-1 / 2}\right) \phi / \zeta-\left(-\delta_{3}\right)^{-1 / 2},
$$

provided that the parameters satisfy the restriction $2+\alpha_{3} \gamma_{3}^{-1 / 2}+\beta_{3}\left(-\delta_{3}\right)^{-1 / 2}=0$ (Lukashevich 1965; Gromak 1977). Hence there exist solutions of $\mathrm{P}_{I I I}$ (4.13) of the form $\phi(\zeta)=\gamma_{3}^{-1 / 2} \Phi_{\zeta}(\zeta) / \Phi(\zeta)$, where $\Phi(\zeta)$ satisfies

$$
\Phi_{\zeta \zeta}+\left(1+\alpha_{3} \gamma_{3}^{-1 / 2}\right) \zeta^{-1} \Phi_{\zeta}+\gamma_{3}^{1 / 2}\left(-\delta_{3}\right)^{-1 / 2} \Phi=0 .
$$

This equation is solvable in terms of Bessel functions or modified Bessel functions depending on the signs of $\gamma_{3}^{1 / 2}$ and $\left(-\delta_{3}\right)^{1 / 2}$. There are essentially four cases, of which we discuss two next.

$\mathrm{P}_{\text {III }}$ possesses the one-parameter family solution,

$$
\phi_{ \pm}(\zeta ; a-1, a-1,1,-1)=\frac{\mathrm{d}}{\mathrm{d} \zeta}\left\{\ln \left[A \zeta^{-\nu} J_{\nu}( \pm \zeta)+B \zeta^{-\nu} Y_{\nu}( \pm \zeta)\right]\right\}
$$

where $\nu=\frac{1}{2}(a-1), J_{\nu}(\zeta)$ and $Y_{\nu}(\zeta)$ are Bessel functions of the first and second kind, with $A$ and $B$ arbitrary constants. Applying the transformation (4.22) with $\epsilon_{3}=\epsilon_{4}=-1$, to (4.27), yields the solution of (4.10) given by

$$
F_{0}\left(\zeta ; 2 \mathrm{i} a,-4,4\left(a^{2}-4\right)\right)=\mathrm{i}\left[2 \zeta \phi_{ \pm}^{2}(\zeta)+a \phi_{ \pm}(\zeta)+2 \zeta\right] / \phi_{ \pm}(\zeta)
$$

We now apply four different versions of the Bäcklund transformation (4.23) to the solution (4.28), taking the lower sign to obtain further solutions rational in both $\zeta$ and $\phi_{-}(\zeta)$, the logarithmic derivative of $\Phi$. This yields the same solution, if $\epsilon_{1}=\epsilon_{2}=\epsilon_{3}=\epsilon_{4}=1$, and

$$
\begin{aligned}
& F_{1}\left(\zeta ;-2 \mathrm{i} a,-4,4\left(a^{2}-16\right)\right) \\
& =\mathrm{i} \frac{(a+4) \zeta^{2} \phi_{-}^{4}+\left(2 a^{2}+4 a-4\right) \zeta \phi_{-}^{3}+\left[2 a \zeta^{2}+\left(a^{3}-a\right)\right] \phi_{-}^{2}+\left(2 a^{2}-4 a-4\right) \zeta \phi_{-}+(a-4) \zeta^{2}}{\left[\zeta \phi_{-}^{2}+(a-1) \phi_{-}+\zeta\right]\left[\zeta \phi_{-}^{2}+(a+1) \phi_{-}+\zeta\right]}, \\
& F_{2}(\zeta ; 6 \mathrm{i}, 4,4(a-2)(a+4))=\mathrm{i} \frac{(a+4) \zeta \phi_{-}^{3}+\left(2 \zeta^{2}+a^{2}+a\right) \phi_{-}^{2}+(3 a+2) \zeta \phi_{-}+2 \zeta^{2}}{\phi_{-}\left[\zeta \phi_{-}^{2}+(a+1) \phi_{-}+\zeta\right]}, \\
& F_{3}(\zeta ;-6 \mathrm{i}, 4,4(a-4)(a+2))=\mathrm{i} \frac{2 \zeta^{2} \phi_{-}^{3}+(3 a-2) \zeta \phi_{-}^{2}+\left(2 \zeta^{2}+a^{2}-a\right) \phi_{-}+(a-4) \zeta}{\zeta \phi_{-}^{2}+(a-1) \phi_{-}+\zeta},
\end{aligned}
$$


for $\epsilon_{1}=\epsilon_{2}=-\epsilon_{3}=-\epsilon_{4}=1, \epsilon_{1}=\epsilon_{2}=-\epsilon_{3}=\epsilon_{4}=1$ and $\epsilon_{1}=\epsilon_{2}=\epsilon_{3}=-\epsilon_{4}=1$, respectively.

An analogous set of solutions may be obtained starting with the one-parameter family solution of $\mathrm{P}_{I I I}$ expressed in terms of modified Bessel functions, namely

$$
\phi_{ \pm}(\zeta ; a-1, a+1,1,-1)=\frac{\mathrm{d}}{\mathrm{d} \zeta}\left\{\ln \left[A \zeta^{-\nu} I_{\nu}( \pm \zeta)+B \zeta^{-\nu} K_{\nu}( \pm \zeta)\right]\right\}
$$

where $\nu=\frac{1}{2}(a-1), I_{\nu}(\zeta)$ and $K_{\nu}(\zeta)$ are Modified Bessel functions, with $A$ and $B$ are arbitrary constants. Applying (4.22) to this solution with $\epsilon_{3}=\epsilon_{4}=-1$ yields

$$
F(\zeta ;-2 \mathrm{i},-4,-4(a-2) a)=\mathrm{i}\left[2 \zeta \phi_{ \pm}(\zeta)+a\right]
$$

while applying it with $\epsilon_{3}=-\epsilon_{4}=-1$ yields

$$
F(\zeta ;-2 \mathrm{i} a, 4,-4(a-2)(a+2))=\mathrm{i}\left[2 \zeta \phi_{ \pm}^{2}(\zeta)+a \phi_{ \pm}(\zeta)-2 \zeta\right] / \phi_{ \pm}(\zeta)
$$

Further solutions can be obtained by applying the Bäcklund transformation (4.23).

All of the solutions of (4.10) so far mentioned have been imaginary. In order to derive real solutions we make use of the property that if $F\left(\zeta ; \mu_{1}, \mu_{2}, K\right)$ is a solution of (4.10) then so is $F\left(\mathrm{i} \zeta ;-\mu_{1},-\mu_{2},-K\right)$. By letting i $a=\alpha$, we obtain a new seed solution for the Bäcklund transformation (4.23) given by $F_{r 1}\left(\zeta ; 2 \alpha, 4,4\left(\alpha^{2}+1\right)\right)= \pm 2 \zeta$, and hence the following real rational solutions to (4.10),

$$
\begin{aligned}
F_{r 2}\left(\zeta ; 2 \alpha, 4,4\left(\alpha^{2}+9\right)\right) & = \pm 2 \zeta \frac{4 \zeta^{2}-4 \alpha \zeta+\alpha^{2}-3}{4 \zeta^{2}-4 \alpha \zeta+\alpha^{2}+1} \\
F_{r 3}\left(\zeta ; 2 \alpha, 4,4\left(\alpha^{2}+25\right)\right) & = \pm 2 \zeta \frac{f_{3}(\zeta)}{g_{3}(\zeta)}
\end{aligned}
$$

where

$$
\begin{aligned}
& f_{3}(\zeta)=64 \zeta^{6}- 192 \alpha \zeta^{5}+48\left(5 \alpha^{2}-3\right) \zeta^{4}-32 \alpha\left(5 \alpha^{2}-7\right) \zeta^{3}+60\left(\alpha^{4}-2 \alpha^{2}-3\right) \zeta^{2} \\
&- 12\left(\alpha^{4}-2 \alpha^{2}-3\right) \alpha \zeta+\alpha^{6}-\alpha^{4}+43 \alpha^{2}+45, \\
& g_{3}(\zeta)=64 \zeta^{6}-192 \alpha \zeta^{5}+48\left(5 \alpha^{2}+1\right) \zeta^{4}-160\left(\alpha^{2}+1\right) \alpha \zeta^{3}+12\left(5 \alpha^{4}+14 \alpha^{2}+9\right) \zeta^{2} \\
&-12\left(\alpha^{4}+6 \alpha^{2}+5\right) \alpha \zeta+\alpha^{6}+11 \alpha^{4}+19 \alpha^{2}+9,
\end{aligned}
$$

and so on. The rational solutions $F_{r 2}(\zeta)$ and $F_{r 3}(\zeta)$ are plotted in Figure 4. We note that Schief (1994) obtained the special case $\alpha=0$ of this hierarchy of rational solutions.

In a similar way the spherical Bessel function solution of order $-\frac{1}{2}$ for $\mathrm{P}_{I I I}$ given by

$$
\phi(\zeta ;-1,-1,1,-1)=[A \cos \zeta-B \sin \zeta] /[A \sin \zeta+B \cos \zeta],
$$

can be used to derive the hyperbolic solution of (4.10),

$$
F_{B 1}(\zeta ; 0,4,16)= \pm \frac{4 \zeta}{\sinh ^{2} \zeta+\cosh ^{2} \zeta}= \pm \frac{8 \zeta \mathrm{e}^{2 \zeta}}{\mathrm{e}^{4 \zeta}+1},
$$

where we have set $A=B$ in order for the solution to be real. This solution can then be used in conjunction with Bäcklund transformation (4.23) to derive the following real solutions of (4.10);

$$
\begin{aligned}
F_{B 2}(\zeta ; 0,4,64) & = \pm \frac{16 \zeta \mathrm{e}^{2 \zeta}\left[(2 \zeta-1) \mathrm{e}^{4 \zeta}-2 \zeta-1\right]}{\mathrm{e}^{8 \zeta}+2\left(8 \zeta^{2}+1\right) \mathrm{e}^{4 \zeta}+1} \\
F_{B 3}(\zeta ; 0,4,144) & = \pm \frac{8 \zeta \mathrm{e}^{2 \zeta}\left[\left(8 \zeta^{2}-12 \zeta+3\right) \mathrm{e}^{8 \zeta}-\left(64 \zeta^{4}+32 \zeta^{2}-6\right) \mathrm{e}^{4 \zeta}+8 \zeta^{2}+12 \zeta+3\right]}{\mathrm{e}^{12 \zeta}+\left(64 \zeta^{4}-64 \zeta^{3}+48 \zeta^{2}+3\right) \mathrm{e}^{8 \zeta}+\left(64 \zeta^{4}+64 \zeta^{3}+48 \zeta^{2}+3\right) \mathrm{e}^{4 \zeta}+1}
\end{aligned}
$$


These solutions to (4.10) are plotted in Figure 5, where it can be seen that this family of solutions are asymptotically zero as $\zeta \rightarrow \pm \infty$.

We can now use these solutions of (4.10) to obtain hierarchies of real solutions of (1.6) by use of the formula, (4.19). Setting $P(z, t)=\frac{1}{2} \int^{z t^{1 / 2}} F(s) s^{-1} \mathrm{~d} s$, we obtain from (4.31),

$$
\begin{aligned}
& P_{r 1}(z, t)= \pm \eta, \\
& P_{r 2}(z, t)= \pm\left\{\eta-2 \tan ^{-1}(2 \eta-a)\right\}, \\
& P_{r 3}(z, t)= \pm \begin{cases}\eta+2 \tan ^{-1} \psi_{1}-2 \tan ^{-1} \psi_{2}-2 \tan ^{-1} \psi_{3}+2 \tan ^{-1}\left(\frac{2 a}{a^{2}-1}\right), & \text { if } a \neq 1, \\
\eta+2 \tan ^{-1} \psi_{4}-2 \tan ^{-1} \psi_{5}-2 \tan ^{-1} \psi_{3}, & \text { if } a=1,\end{cases}
\end{aligned}
$$

where $\eta=z t^{1 / 2}$ and

$$
\begin{aligned}
& \psi_{1}=\frac{8}{3\left(a^{2}+1\right)} \eta^{5}-\frac{8 a}{\left(a^{2}+1\right)} \eta^{4}+\frac{4\left(a^{2}+7\right)}{3\left(a^{2}+1\right)} \eta^{3}-16 a \eta^{2}+\frac{1}{2}\left(3 a^{2}+7\right) \eta-\frac{1}{6}\left(a^{2}+7\right) a \\
& \psi_{2}=\frac{2}{3} \eta^{3}-\frac{4}{3} a \eta^{2}+\frac{5}{6}\left(a^{2}+1\right) \eta-\frac{1}{6} a^{3}-\frac{7}{6} a, \\
& \psi_{3}=\frac{2}{3} \eta-\frac{1}{3} a \\
& \psi_{4}=\frac{4}{3} \eta^{5}-4 \eta^{4}+\frac{16}{3} \eta^{3}-\frac{16}{3} \eta^{2}+5 \eta-\frac{4}{3}, \\
& \psi_{5}=\frac{2}{3} \eta^{3}-\frac{4}{3} \eta^{2}+\frac{5}{3} \eta-\frac{4}{3},
\end{aligned}
$$

Similarly, we obtain from the family (4.32),

$$
\begin{aligned}
P_{B 1}(z, t)= & \pm \tan ^{-1}\{\sinh (2 \eta)\} \\
P_{B 2}(z, t)= & \pm\{\ln [\exp (4 \eta)+4 \mathrm{i} \eta \exp (2 \eta)+1]-\ln [\exp (4 \eta)-4 \mathrm{i} \eta \exp (2 \eta)+1]\} \\
P_{B 3}(z, t)= \pm \mathrm{i}\left\{\ln \left[\exp (6 \eta)+\mathrm{i}\left(8 \eta^{2}-4 \eta+1\right) \exp (4 \eta)+\left(8 \eta^{2}+4 \eta+1\right) \exp (2 \eta)+\mathrm{i}\right]\right. & \\
& \left.\quad-\ln \left[\exp (6 \eta)-\mathrm{i}\left(8 \eta^{2}-4 \eta+1\right) \exp (4 \eta)+\left(8 \eta^{2}+4 \eta+1\right) \exp (2 \eta)-\mathrm{i}\right]\right\},
\end{aligned}
$$

where $\eta(z, t)=z t^{1 / 2}$. We note again that the expressions (4.34) are real, despite their closed form expressions involving the complex number i. In Figure 6, we plot the solution $u(x, y, t)=P_{B 3}(x+y, t)+P_{B 3}(x-y, t)$ for $t=1,4$ and 25. In Figure 7, we plot the solution $u(x, y, t)=P_{B 3}(x+y, t)+P_{r 3}(x-y, t)$ for $a=1$ and $t=1$.

Case 4.5(ii) $\mu_{1} \neq 0, \mu_{2}=0$. Given $\phi(\zeta)$ and $\tilde{\phi}(\tilde{\zeta})$ satisfying $\mathrm{P}_{I I I}$ (4.13) with constants $\alpha_{3}=2 \mathrm{i} \mu_{1} / \nu, \beta_{3}=\frac{1}{8} \nu\left(4+\mathrm{i} K / \mu_{1}\right), \gamma_{3}=0$ and $\delta_{3}=-\frac{1}{4} \nu^{2}$, and $\tilde{\alpha}_{3}=2 \mathrm{i} \tilde{\mu}_{1} / \tilde{\nu}, \tilde{\beta}_{3}=\frac{1}{8} \tilde{\nu}\left(4+\mathrm{i} \tilde{K} / \tilde{\mu}_{1}\right)$, $\tilde{\gamma}_{3}=0$ and $\tilde{\delta}_{3}=-\frac{1}{4} \tilde{\nu}^{2}$, respectively, with $\nu \neq 0$ and $\tilde{\nu} \neq 0$, we have,

$$
\begin{aligned}
& u(x, y, t)=\frac{1}{2} \mathrm{i} \int^{\zeta}\left[\frac{\phi_{\zeta_{1}}\left(\zeta_{1}\right)-\frac{1}{2} \nu}{\phi\left(\zeta_{1}\right)}\right] \mathrm{d} \zeta_{1}+\frac{1}{2} \mathrm{i} \int^{\tilde{\zeta}}\left[\frac{\tilde{\phi}_{\tilde{\zeta}_{1}}\left(\tilde{\zeta}_{1}\right)-\frac{1}{2} \tilde{\nu}}{\tilde{\phi}\left(\tilde{\zeta}_{1}\right)}\right] \mathrm{d} \tilde{\zeta}_{1}, \\
& v(x, y, t)=-\frac{1}{4} \int^{\zeta} \int^{\zeta_{1}}\left[\frac{\phi_{\zeta_{1}}\left(\zeta_{1}\right)-\frac{1}{2} \nu}{\phi\left(\zeta_{1}\right)}\right]^{2} \mathrm{~d} \zeta_{2} \mathrm{~d} \zeta_{1}-\frac{1}{4} \int^{\tilde{\zeta}} \int^{\tilde{\zeta}_{1}}\left[\frac{\tilde{\phi}_{\tilde{\zeta}_{2}}\left(\tilde{\zeta}_{2}\right)-\frac{1}{2} \tilde{\nu}}{\tilde{\phi}_{(}\left(\tilde{\zeta}_{2}\right)}\right]^{2} \mathrm{~d} \tilde{\zeta}_{2} \mathrm{~d} \tilde{\zeta}_{1},
\end{aligned}
$$

where $\zeta=(x+y) t^{1 / 2}$ and $\tilde{\zeta}=(x-y) t^{1 / 2}$. The simplest example of such a solution of $\mathrm{P}_{I I I}$ is given by $\phi(\zeta)=\epsilon \zeta^{1 / 3}$ and $\tilde{\phi}(\tilde{\zeta})=\epsilon \tilde{\zeta}^{1 / 3}$, in which case we have the solution for (1.6), where $\kappa_{1}$ is determined by the parameters $\nu, \epsilon$ and $\mu_{1}$,

$$
\begin{aligned}
& u(x, y, t)=\frac{1}{6} \mathrm{i} \ln (\zeta \tilde{\zeta})+\kappa_{1}\left(\zeta^{2 / 3}+\tilde{\zeta}^{2 / 3}\right), \\
& v(x, y, t)=\frac{1}{36} \ln (\zeta \tilde{\zeta})-\mathrm{i} \kappa_{1}\left(\zeta^{2 / 3}+\tilde{\zeta}^{2 / 3}\right)+\kappa_{1}^{2}\left(\zeta^{4 / 3}+\tilde{\zeta}^{4 / 3}\right) .
\end{aligned}
$$


A Bäcklund transformation for $\mathrm{P}_{I I I}$ with $\gamma \equiv 0$ is given in equations $(1.2 \mathrm{a}, \mathrm{b})$ of Milne \& Clarkson (1993). Using this Bäcklund transformation on the "seed" solution $\phi(\zeta)=\epsilon \zeta^{1 / 3}$, one can obtain a hierarchy of solutions of $\mathrm{P}_{I I I}$ with $\gamma=0$ which are rational in $\zeta^{1 / 3}$. We leave it to the reader to calculate the corresponding expressions for $u(x, y, t)$ and $v(x, y, t)$, and the "lift" of the Bäcklund transformation for $\mathrm{P}_{I I I}$ with $\gamma=0$ to the family of solutions (4.35) of (1.6).

Case 4.5(iii) $\mu_{1}=\mu_{2}=0 . \quad$ Given $\phi\left(\zeta ; 1,-\frac{1}{4} K, 0,0\right)$ and $\tilde{\phi}\left(\tilde{\zeta} ; 1,-\frac{1}{4} \tilde{K}, 0,0\right)$ satisfying $\mathrm{P}_{I I I}(4.13)$, we have,

$$
\begin{aligned}
& u(x, y, t)=\frac{1}{2} \mathrm{i} \ln [\phi(\zeta) \tilde{\phi}(\tilde{\zeta})], \\
& v(x, y, t)=-\frac{1}{4} \int^{\zeta} \int^{\zeta_{1}}\left[\frac{\phi^{\prime}\left(\zeta_{2}\right)}{\phi\left(\zeta_{2}\right)}\right]^{2} \mathrm{~d} \zeta_{2} \mathrm{~d} \zeta_{1}-\frac{1}{4} \int^{\tilde{\zeta}} \int^{\tilde{\zeta}_{1}}\left[\frac{\tilde{\phi}^{\prime}\left(\tilde{\zeta}_{2}\right)}{\tilde{\phi}\left(\tilde{\zeta}_{2}\right)}\right]^{2} \mathrm{~d} \tilde{\zeta}_{2} \mathrm{~d} \tilde{\zeta}_{1}
\end{aligned}
$$

where $\zeta=(x+y) t^{1 / 2}$ and $\tilde{\zeta}=(x-y) t^{1 / 2}$. An alternative expression for $v$ is given by,

$$
v(x, y, t)=-\frac{1}{2} \int^{\zeta}\left[\phi\left(\zeta_{1}\right)+\frac{K}{4 \phi\left(\zeta_{1}\right)}\right] \frac{\mathrm{d} \zeta_{1}}{\zeta_{1}}-\frac{1}{2} \int^{\tilde{\zeta}}\left[\tilde{\phi}\left(\tilde{\zeta}_{1}\right)+\frac{\tilde{K}}{4 \tilde{\phi}\left(\tilde{\zeta}_{1}\right)}\right] \frac{\mathrm{d} \zeta_{1}}{\tilde{\zeta}_{1}} .
$$

In this case when $K=0$, the solution of $\mathrm{P}_{I I I}$ is

$$
\phi(z)=\frac{\kappa_{1} z^{\lambda-1}}{\left(z^{\lambda}-\frac{1}{4} \kappa_{1} \lambda^{-4}\right)^{2}}, \quad \phi(z)=\frac{2}{z\left(\ln z+\kappa_{1}\right)^{2}}
$$

(cf., Airault 1979). We leave it to the reader to calculate the associated solutions for (1.6).

\section{Further solution families}

In this section we apply a known Bäcklund transformation for the system (1.6) to two of the simplest of the solutions found in the families (4.15). The result is new families of solutions involving the arguments and moduli of sums of products of exponential and Bessel function expansions with arbitrary coefficients, respectively. The analysis for the other exact solutions described in the previous section will be similar.

The Bäcklund transformation for the system (1.6) we employ can be found in Dubrovsky \& Konopelchenko (1993), Konopelchenko et al. (1992) and Nimmo (1993). Let $u, v$ be a solution of (1.6). If $\psi$ is a solution of

$$
\psi_{x y}-U \psi=0, \quad U=u_{x} u_{y}-\mathrm{i} u_{x y},
$$

then a new solution of (1.6) is given by,

$$
\tilde{u}=u+\mathrm{i} \ln (\psi / \bar{\psi}), \quad \tilde{v}=v+\ln (\psi \bar{\psi}) .
$$

For the solution found in (4.15), we have

$$
U=\left[\tilde{\eta}\left(\tilde{\zeta} ; \mu_{1}, 1, \beta\right)\right]^{2}+\tilde{\eta}_{\tilde{\zeta}}\left(\tilde{\zeta} ; \mu_{1}, 1, \beta\right)-\left[\eta\left(\zeta ; \mu_{1}, 1, c\right)\right]^{2}-\eta_{\zeta}\left(\zeta ; \mu_{1}, 1, c\right),
$$

where $\eta\left(\zeta ; \mu_{1}, \mu_{2}, c\right)$ and $\tilde{\eta}\left(\tilde{\zeta} ; \mu_{1}, \mu_{2}, \beta\right)$ satisfy (4.7) with $\zeta=x+y-c t$ and $\tilde{\zeta}=x-y-\beta t$. If we write, $q(\zeta)=\left[\eta\left(\zeta ; \mu_{1}, 1, c\right)\right]^{2}+\eta_{\zeta}\left(\zeta ; \mu_{1}, 1, c\right)$ and $\tilde{q}(\tilde{\zeta})=\left[\tilde{\eta}\left(\tilde{\zeta} ; \mu_{1}, 1, \beta\right)\right]^{2}+\tilde{\eta}_{\tilde{\zeta}}\left(\tilde{\zeta} ; \mu_{1}, 1, \beta\right)$, then we can rewrite (5.1) as,

$$
\psi_{\zeta \zeta}-q(\zeta) \psi=\psi_{\tilde{\zeta} \tilde{\zeta}}-\tilde{q}(\tilde{\zeta}) \psi
$$

It is a curious fact that if $f(r, s)=\psi_{r r}-q(r) \psi=\psi_{s s}+\tilde{p}(s) \psi$, then $f(r, s)$ satisfies the same equation as does $\psi$. Thus, if we can solve the eigenvalue problems for the ordinary differential equations,

$$
\phi_{\zeta \zeta}-q(\zeta) \phi=\lambda \phi, \quad \tilde{\phi}_{\tilde{\zeta} \tilde{\zeta}}-\tilde{q}(\tilde{\zeta}) \tilde{\phi}=\lambda \tilde{\phi}
$$


we can set $\psi(\zeta, \tilde{\zeta})=\phi(\zeta ; \lambda) \tilde{\phi}(\tilde{\zeta} ; \lambda)$. However we can go further; we can obtain solutions of (5.1) written as formal sums of eigenfunction expansions,

$$
\psi(\zeta, \tilde{\zeta})=\sum_{\lambda} C_{\lambda} \phi(\zeta ; \lambda) \tilde{\phi}(\tilde{\zeta} ; \lambda)
$$

where $C_{\lambda}$ is a constant and the $\psi_{i, \lambda}$ satisfy (5.3) for each given $\lambda$, since the governing equation for $\psi,(5.1)$, is linear.

As an example, we apply the Bäcklund transformation (5.2) to the "seed" solution, (4.16) which is obtained from the solution $Q(z ; 1)=1 / z$ of $(4.7)$ with $\mu_{2}=1$, or from $Q=0$. Then $p(s)=0=\tilde{p}(s)$, and the eigenvalue problem becomes

$$
\phi_{i, z z}-\lambda \phi_{i}=0, \quad \text { for } \quad i=1,2 .
$$

If $\lambda=\mu^{2}$, then this has solution $\phi_{i}(z)=A_{\mu} \exp (\mu \zeta)+B_{\mu} \exp (-\mu \zeta)$, with $A_{\mu}$ and $B_{\mu}$ arbitrary constants, and so

$$
\psi(\zeta, \tilde{\zeta})=\sum_{\mu}\left[A_{\mu} \exp (\mu \zeta)+B_{\mu} \exp (-\mu \zeta)\right]\left[\tilde{A}_{\mu} \exp (\mu \tilde{\zeta})+\tilde{B}_{\mu} \exp (-\mu \tilde{\zeta})\right]
$$

If $\lambda=-\mu^{2}$, then $\phi_{i}(z)=A_{\mu} \sin (\mu \zeta)+B_{\mu} \cos (\mu \zeta)$, where $A_{\mu}$ and $B_{\mu}$ are constants, and so

$$
\psi(\zeta, \tilde{\zeta})=\sum_{\mu} C_{\mu} \sin \left(\mu \zeta+\delta_{\mu}\right) \sin \left(\mu \tilde{\zeta}+\tilde{\delta}_{\mu}\right)
$$

with $C_{\mu}, \delta_{\mu}$ and $\tilde{\delta}_{\mu}$ arbitrary constants.

Applying the Bäcklund transformation (5.2) to the solution (4.17) obtained from the solution $y(x ; 2)=2\left(x^{3}-2\right) /\left[x\left(x^{3}+4\right)\right]$ of $\mathrm{P}_{I I}(4.8)$ yields the eigenvalue problem

$$
\phi_{i, z z}+\left(\mu^{2}-2 z^{-2}\right) \phi_{i}=0, \quad i=1,2 .
$$

This has solution $\phi_{i}(z)=z^{1 / 2}\left[A_{\mu} J_{\nu}(\mu z)+B_{\mu} J_{-\nu}(\mu z)\right]$, where $A_{\mu}$ and $B_{\mu}$ are constants and $J_{\nu}(x)$ is the usual Bessel function, with $\nu=\frac{1}{2} \sqrt{5}$, and so

$$
\psi(\zeta, \tilde{\zeta})=\sum_{\mu}(\zeta \tilde{\zeta})^{1 / 2}\left[A_{\mu} J_{\nu}(\mu \zeta)+B_{\mu} J_{-\nu}(\mu \zeta)\right]\left[\tilde{A}_{\mu} J_{\nu}(\mu \tilde{\zeta})+\tilde{B}_{\mu} J_{-\nu}(\mu \tilde{\zeta})\right]
$$

It is clear that it is possible to obtain many further exact solutions for (1.6) using solutions obtained in $\S 4$ above in conjunction with the Bäcklund transformation (5.2). We shall not pursue this further here.

\section{Painlevé analysis and integrability of the generalized Maxwell-Bloch system}

The Painlevé Conjecture (or Painlevé ODE test) as formulated by Ablowitz et al. (1978, 1980), asserts that every ordinary differential equation which arises as a symmetry reduction of a completely integrable nonlinear partial differential equation is of Painlevé type, though perhaps only after a transformation of variables. Subsequently, Weiss et al. (1983), proposed the Painlevé PDE test as a method of applying the Painlevé ODE test directly to a given partial differential equation without having to consider symmetry reductions (which might not exist). Despite being by no means foolproof the Painlevé tests appear to provide a useful criterion for the identification of completely integrable partial differential equations. 


\subsection{Painlevé analysis of the Sine-Gordon system (1.6)}

Here we discuss the integrability of the generalization of the Sine-Gordon system (1.6) given by

$$
\begin{aligned}
& u_{x y t}+\alpha u_{x} v_{y t}+\beta u_{y} v_{x t}=0 \\
& v_{x y}-u_{x} u_{y}=0
\end{aligned}
$$

where $\alpha$ and $\beta$ are arbitrary constants. To apply the Painlevé PDE test due to Weiss et al. (1983), to the system (6.1) we seek a solution in the form

$$
u(x, y, t)=\sum_{k=0}^{\infty} u_{k}(y, t) \phi^{k+p}(x, y, t), \quad v(x, y, t)=\sum_{k=0}^{\infty} v_{k}(y, t) \phi^{k+q}(x, y, t),
$$

where $\phi(x, y, t)=x+\psi(y, t)$, with $\psi(y, t)$ an arbitrary analytic function, and $u_{k}(y, t), v_{k}(y, t)$, $k=0,1,2, \ldots$, are analytic functions such that $u_{0} v_{0} \not \equiv 0$, in the neighbourhood of an arbitrary, non-characteristic movable singularity manifold defined by $\phi(x, y, t)=0$, and $p$ and $q$ are constants to be determined. By leading order analysis we find that $p=q=0$. Thus the standard procedure for the applying the Painlevé PDE test to the system (6.1) needs to be modified. Since only the derivatives of $u$ and $v$ arise in (6.1), then, following Clarkson (1986b), we seek a solution in the form

$$
\begin{aligned}
& u(x, y, t)=u_{00}(y, t) \ln \phi(x, y, t)+\sum_{k=0}^{\infty} u_{k}(y, t) \phi^{k}(x, y, t), \\
& v(x, y, t)=v_{00}(y, t) \ln \phi(x, y, t)+\sum_{k=0}^{\infty} v_{k}(y, t) \phi^{k}(x, y, t),
\end{aligned}
$$

where the same notation applies as in $(6.2)$ and $u_{00}(y, t)$ and $v_{00}(y, t)$ are analytic functions to be determined. By leading order analysis, from the coefficients of $\phi^{-3}$, we find that $u_{00}(y, t)=$ $\mathrm{i} \sqrt{2 /(\alpha+\beta)}$ and $v_{00}(y, t)=2 /(\alpha+\beta)$. Equating the coefficients of powers of $\phi^{r-3}$ yields the general recursion relation

$$
\mathbf{Q}(r)\left(\begin{array}{c}
u_{r} \\
v_{r}
\end{array}\right) \equiv\left(\begin{array}{cc}
r^{2}(r-3) \psi_{y} \psi_{t} & \sqrt{2(\alpha+\beta)} \mathrm{i} r(r-1) \psi_{y} \psi_{t} \\
2 \sqrt{2} \mathrm{i} r \psi_{y} / \sqrt{\alpha+\beta} & r(r-1) \psi_{y}
\end{array}\right)\left(\begin{array}{l}
u_{r} \\
v_{r}
\end{array}\right)=\left(\begin{array}{c}
F_{r} \\
G_{r}
\end{array}\right),
$$

where $F_{r}$ and $G_{r}$ are known functions of $u_{0}, v_{0}, \ldots, u_{r-1}, v_{r-1}, \psi$ and their derivatives. These recursion relations uniquely define $u_{r}, v_{r}$ unless

$$
\operatorname{det} \mathbf{Q}(r)=(r+1) r^{2}(r-1)(r-4) \psi_{y}^{2} \psi_{t}=0 .
$$

Therefore the resonances are $r=-1,0,1,4$. The resonance $r=-1$ corresponds to the fact that $\psi(y, t)$ is an arbitrary function and the double resonance $r=0$ to both $u_{0}(y, t)$ and $v_{0}(y, t)$ being arbitrary functions.

For the resonance $r=1$, there is one equation whose solution yields $u_{1}=-u_{0, y} /\left(2 \psi_{y}\right)$. Substituting this into the second equation yields the following "compatibility condition",

$$
(\alpha-\beta)\left(\sqrt{2} \psi_{y t}+\mathrm{i} \sqrt{\alpha+\beta} u_{0, y} \psi_{t}\right)=0 .
$$

Since $\psi$ and $u_{0}$ are arbitrary, this is identically satisfied if and only if $\alpha=\beta$, in which case $v_{1}$ is another arbitrary function. Otherwise, if $\alpha \neq \beta$, then it is necessary to introduce $u_{11}(t) \phi(x, t) \ln \phi(x, t)$ and $v_{11}(t) \phi(x, t) \ln \phi(x, t)$ terms, where $u_{11}(t)$ and $v_{11}(t)$ are to be determined, into the expansion (6.3) and at higher orders of $\phi(x, t)$, higher and higher powers of $\ln \phi(x, t)$ are required; a strong indication of non-Painlevé behaviour. 
If $\alpha=\beta$, then at the resonance $r=4$ there is only one independent equation and so either $u_{4}$ or $v_{4}$ is arbitrary. Hence we can indeed obtain an expansion for $u, v$ in the form (6.3) with $u_{0}$, $v_{0}, v_{1}$ and $u_{4}$ (or $v_{4}$ ), arbitrary functions in the case when $\alpha=\beta$; since the expressions obtained are complex, we do not reproduce details here. Therefore the Painlevé PDE test suggests that the Sine-Gordon system (1.6) is the only integrable case of the system (6.1).

\subsection{Integrability of the generalized, real, pumped Maxwell-Bloch equation}

The integrability condition of the generalized, real, pumped Maxwell-Bloch system $(3.4 a, b)$ is

$$
w w_{z z t}-w_{z} w_{z t}-m_{1}(t) w_{z}+w^{3} w_{t}-\frac{1}{2} \frac{\mathrm{d} m_{2}}{\mathrm{~d} t} w^{2}=0
$$

where $m_{1}(t)$ and $m_{2}(t)$ are arbitrary functions. It is routine to apply Painlevé analysis to (6.5) and show that it satisfies the necessary conditions of the Painlevé PDE test due to Weiss et al. (1983) to be completely integrable.

As mentioned in $\S 4.1$ above, the sine-Gordon equation (1.2) is equivalent to the special case of (6.5) with $m_{1}(t) \equiv 0$ and $m_{2}(t) \equiv 0$, whilst the real, pumped Maxwell-Bloch system (4.1) is equivalent to the special case with $m_{1}(t) \equiv 0$. The ubiquitous sine-Gordon equation (1.2) is one of the fundamental soliton equations solvable by inverse scattering using the AKNS method (Ablowitz et al. 1974). However, the Lax pair for the complex pumped Maxwell-Bloch system is non-isospectral (Burtsev et al. 1987).

It can be shown that a non-isospectral Lax pair associated with (6.5) is given by

$$
\begin{aligned}
& \boldsymbol{\psi}_{z}=\left\{-\mathrm{i} \lambda \boldsymbol{\sigma}_{3}-\frac{1}{2} \mathrm{i} w \boldsymbol{\sigma}_{2}\right\} \boldsymbol{\psi}, \\
& \boldsymbol{\psi}_{t}=\left\{\mathrm{i} \frac{w_{z t}+m_{1}(t)}{4 \lambda w} \boldsymbol{\sigma}_{3}+\frac{\mathrm{i} w_{t}}{4 \lambda} \boldsymbol{\sigma}_{1}+\frac{\mathrm{i} m_{1}(t)}{8 \lambda^{2}} \boldsymbol{\sigma}_{2}\right\} \boldsymbol{\psi}=0 .
\end{aligned}
$$

where $\boldsymbol{\sigma}_{1}, \boldsymbol{\sigma}_{2}$ and $\boldsymbol{\sigma}_{3}$ are the Pauli spin matrices given by

$$
\boldsymbol{\sigma}_{1}=\left(\begin{array}{rr}
0 & 1 \\
1 & 0
\end{array}\right), \quad \sigma_{2}=\left(\begin{array}{rr}
0 & -\mathrm{i} \\
\mathrm{i} & 0
\end{array}\right), \quad \boldsymbol{\sigma}_{3}=\left(\begin{array}{rr}
1 & 0 \\
0 & -1
\end{array}\right)
$$

Equations $(6.6 a, b)$ are compatible, i.e. $\boldsymbol{\psi}_{z t}=\boldsymbol{\psi}_{t z}$ provided that $w(z, t)$ satisfies $(6.5)$ and $\lambda$ satisfies

$$
\lambda^{2}(t)=-\frac{1}{4} m_{2}(t)+\lambda_{0}^{2}
$$

where $\lambda_{0}$ is a constant. Hence if $\frac{\mathrm{d} m_{2}}{\mathrm{~d} t} \neq 0$, then the Lax Pair (6.6) is non-isospectral. We remark that the Lax pair (6.6) reduces to that for the sine-Gordon equation (1.2) if $m_{1}(t)=m_{2}(t)=0$ and that for the real, pumped Maxwell-Bloch system (4.1) if $m_{1}(t)=0$. Further we note that the spectral problem $(6.6 a)$ is the standard AKNS spectral problem whilst if $m_{1}(t) \neq 0$, then $(6.6 b)$ involves powers of both $\lambda^{-1}$ and $\lambda^{-2}$.

Thus, either there exists an isospectral Lax pair for (6.5), or the definition of integrability must be extended to include non-isospectral Lax pairs. Either way, it appears that the (6.5) can be viewed as an example of an equation on the "edge" of integrability; it arises as a reduction of an integrable equation, it possesses the Painlevé property yet only a non-isospectral Lax pair is currently known.

\section{Discussion}

Using the nonclassical reduction method due to Bluman \& Cole (1969), and a Bäcklund transformation (5.2), we are able to generate many families of exact solutions for the system (1.6). 
Further, the reductions obtained have important consequences for the integrability of the pumped Maxwell-Bloch system, and the numerical study of (1.6), as illustrated by Figure 3. The difficulty with using numerics is in the fact that every variable can be scaled arbitrarily and the result will still be a solution of the system; this is the content of the classical symmetry group for the system. Any numerical scheme for this equation must be able to fix the scaling on each variable, otherwise a purely artifactual "chaos" will be observed.

There are two final points we wish to note. Firstly, the Bäcklund transformation (5.2) for the system (1.6) appears to have no relation to the Bäcklund transformations discovered for the various families of exact solutions written down in $\S 4$. Secondly, there now exist several examples where the nonclassical method has found a solution in the form of a decoupled sum of solutions of a lower dimensional equation (cf. Clarkson \& Mansfield 1994b; Mansfield \& Clarkson 1994), a fact which is interesting in itself. Also of interest is the fact that there may exist families of solutions in which the pieces of the decoupled sum interact with each other. Indeed, for the shallow water wave equation discussed in Clarkson \& Mansfield (1994b), the nonclassical method obtained decoupled two-soliton solutions in terms of solutions of the first Painlevé equation. The simplest examples of these two-solitons are part of a larger family of interacting two-solitons obtained by the Hirota method and by the singularity manifold method, but not by the nonclassical method. Conversely, neither the Hirota nor the singularity manifold methods appear to find the more general decoupled solution. It would be of great interest if a similar phenomenon were to be found for the system (1.6).

Acknowledgement. We thank Mark Ablowitz and Colin Rogers, for helpful comments and stimulating discussions. We also thank the Program in Applied Mathematics, University of Colorado at Boulder, for their hospitality during our visit whilst some of this work was done. The research of PAC and ELM is supported by EPSRC (grant GR/H39420) and that of AEM by an EPSRC Research Studentship, which are gratefully acknowledged.

\section{References}

Ablowitz, M.J. \& Clarkson, P.A. 1991 "Solitons, Nonlinear Evolution Equations and Inverse Scattering," L.M.S. Lect. Notes Math., 149, C.U.P., Cambridge.

Ablowitz, M.J. \& Haberman, R. 1975 Nonlinear evolution equations - two and three dimensions Phys. Rev. Lett. 35 1185-1188.

Ablowitz, M.J., Kaup, D.J. \& Newell, A.C. 1974 Coherent pulse propagation, a dispersive, irreversible phenomenon J. Math. Phys. 15 1852-1858.

Ablowitz, M.J., Kaup, D.J., Newell, A.C. \& Segur, H. 1974 The inverse scattering transform Fourier analysis for nonlinear problems Stud. Appl. Math. 53 249-315.

Ablowitz, M.J., Ramani, A. \& Segur, H. 1978 Nonlinear evolution equations and ordinary differential equations of Painlevé type Phys. Rev. Lett. 23 333-338.

Ablowitz, M.J., Ramani, A. \& Segur, H. 1980 A connection between nonlinear evolution equations and ordinary differential equations of P-type. I J. Math. Phys. 21 715-721.

Ablowitz, M.J. \& Segur H. 1981 "Solitons and the Inverse Scattering Transform," SIAM, Philadelphia.

Airault, H. 1979 Rational solutions of Painlevé equations Stud. Appl. Math. 61 31-53.

Athorne, C. \& Nimmo, J.J.C. 1991 On the Moutard transformation for integrable partial differential equations Inverse Problems 7 809-826.

Bluman, G.W. \& Cole, J.D. 1969 The general similarity solution of the heat equation J. Math. Mech. 18 1025-1042.

Boiti, M., Leon, J.J.-P., Martina, L. \& Pempinelli, F. 1988 Scattering of localized solitons in the plane Phys. Lett. 132A 432-439. 
Bureau, F. 1972 Equations différentielles du second ordre en $\ddot{y}$ et du second degré en $y$ dont l'intégrale générale est à points critiques fixès Ann. Mat. Pura Appl. (IV) 91 163-281.

Burtsev, S.P. 1993 The Maxwell-Bloch system with pumping and the fifth Painlevé equation Phys. Lett. 177A 341-344.

Burtsev, S.P. \& Gabitov, I.R. 1994 Alternative integrable equations of nonlinear optics Phys. Rev. A49 2065-2070.

Burtsev, S.P., Mikhailov, A.V. \& Zakharov, V.E. 1987 Inverse scattering method with variable spectral parameter Theor. Math. Phys. 70 227-240.

Champagne, B., Hereman, W. \& Winternitz, P. 1991 The computer calculation of Lie point symmetries of large systems of differential equations Comp. Phys. Comm. 66 319-340.

Clarkson, P.A. $1986 a$ Remarks on the two-dimensional Sine-Gordon equation and the Painlevé tests Lett. Math. Phys. 10 297-299.

Clarkson, P.A. $1986 b$ The Painlevé property, a Modified Boussinesq equation and a Modified Kadomtsev-Petviashvili equation Physica 19D 447-450.

Clarkson, P.A. 1990 New exact solutions of the Boussinesq equation Europ. J. Appl. Math. 1 279-300.

Clarkson, P.A. \& Mansfield, E.L. 1994a Symmetry reductions and exact solutions of a class of nonlinear heat equations Physica 70D 250-288.

Clarkson, P.A. \& Mansfield, E.L. $1994 b$ On a shallow water wave equation Nonlinearity 7 975-1000.

Clarkson, P.A. \& Mansfield, E.L. $1994 c$ Algorithms for the nonclassical method of symmetry reductions SIAM J. Appl. Math $\mathbf{5 4}$ to appear.

Cosgrove, C.M. \& Scoufis, G. 1993 Painlevé classification of a class of differential equations of the second order and second degree Stud. Appl. Math. 88 25-87.

Darboux, G. 1887-1896 Leçons sur la théorie générale des surfaces et les applications géométrique du calcul infinitésimal, t. 1-4, Paris.

Dubrovsky, V.G. \& Konopelchenko, B.G. 1993 The 2+1-dimensional integrable generalization of the sine-Gordon equations: II. Localized solutions Inverse Problems 9 391-416.

Fokas, A.S. \& Ablowitz, M.J. 1982 On a unified approach to transformations and elementary solutions of Painlevé equations J. Math. Phys. 23 2033-2042.

Fokas, A.S. \& Ablowitz, M.J. 1984 On the inverse scattering transform of multidimensional nonlinear evolution equations related to first-order systems in the plane J. Math. Phys. 25 2494-2505.

Fokas, A.S. \& Santini, P.M. 1989 Coherent structures in multidimensions Phys. Rev. Lett. 63 $1329-1333$.

Fokas, A.S. \& Santini, P.M. 1990 Dromions and a boundary value problem for the Davey-Stewartson I equation Physica 44D 99-130.

Gibbon, J.D., Newell, A.C., Tabor, M. \& Zeng, Y.B. 1988 Lax pairs, Bäcklund transformations and special solutions for ordinary differential equations Nonlinearity 1 481-490.

Gromak, V.I. 1975 Theory of Painlevé's equation Diff. Eqns. 11 285-287.

Gromak, V.I. 1977 One-parameter systems of solutions of Painlevé's equations Diff. Eqns. 14 15101513.

Hereman, W. 1994 Review of symbolic software for the computation of Lie symmetries of differential equations Euromath Bull. 1, no. 2, 45-79.

Ince, E.L. 1956 "Ordinary Differential Equations," Dover, New York.

Johnson, M.E., Rogers, C., Schief, W.K. \& Seiler, W.M. 1994 On moving pseudospherical surfaces: a generalised Weingarten system and its formal analysis Lie Groups and their Applications 1 $124-136$.

Kitaev, A.V., Law, C.K. \& McLeod, J.B. 1994 Rational solutions of the fifth Painlevé equation Diff. Int. Eqns. 7 967-1000. 
Kitaev, A.V., Rybin, A.V. \& Timonen, J. 1993 Similarity solutions of the deformed Maxwell-Bloch system J. Phys. A: Math. Gen. 26 3583-3595.

Konopelchenko, B.G. 1993 "Solitons in Multi-dimensions," World Scientific, Singapore.

Konopelchenko, B.G. \& Dubrovsky, V.G. 1993 The 2+1-dimensional integrable generalization of the sine-Gordon equations: I. $\partial-\bar{\partial}$-dressing and the initial value problem Stud. Appl. Math. 90 189-223.

Konopelchenko, B.G. \& Rogers, C. 1991 On $2+1$-dimensional nonlinear systems of Loewner type Phys. Lett. 158A 391-397.

Konopelchenko, B.G. \& Rogers, C. 1993 On generalized Loewner systems: novel integrable equations in $2+1$-dimensional J. Math. Phys. 34 214-242.

Konopelchenko, B.G., Schief, W. \& Rogers, C. 1992 A (2+1)-dimensional sine-Gordon system: its auto-Bäcklund transformation Phys. Lett. 172A 39-48.

Levi, D. \& Winternitz, P. 1989 Nonclassical symmetry reduction: example of the Boussinesq equation J. Phys. A: Math. Gen. 22 2915-2924.

Loewner, C. 1952 Generation of solutions of systems of partial differential equations by composition of infinitesimal Bäcklund transformations J. Anal. Math. 2 219-242.

Lukashevich, N.A. 1965 Elementary solutions of certain Painlevé equations Diff. Eqns. 1 561-564.

Mansfield, E.L. 1993 "diffgrob2: A symbolic algebra package for analysing systems of PDE using MAPLE", ftp euclid.exeter.ac.uk, login: anonymous, password: your email address, directory: pub/liz.

Mansfield, E.L. \& Clarkson, P.A. 1994 Applications of the differential algebra package diffgrob2 to reductions of PDE in "Proceedings of the Fourteenth IMACS World Congress on Computation and Applied Mathematics," (Ed. W.F. Ames) Georgia Inst. Tech., Vol. 1, pp336-339.

Milne, A.E. \& Clarkson, P.A. 1993 Rational solutions and Bäcklund transformations for the third Painlevé equation in "Applications of Analytic and Geometric Methods to Nonlinear Differential Equations," (Ed. P.A. Clarkson) NATO ASI Series C: Mathematical and Physical Sciences, 413, Kluwer, Dordrecht, pp341-352.

Murato, Y. 1985 Rational solutions of the second and fourth Painlevé equations Funkcial. Ekvacioj 28 1-32.

Nimmo, J.J.C. 1992 A class of solutions of the Konopelchenko-Rogers equations Phys. Lett. 168A $113-119$.

Nimmo, J.J.C. 1993 Darboux transformations in $(2+1)$-dimensions in "Applications of Analytic and Geometric Methods to Nonlinear Differential Equations," (Ed. P.A. Clarkson) NATO ASI Series C: Mathematical and Physical Sciences, 413, Kluwer, Dordrecht, pp183-192.

Okamoto, K. 1987 Studies on the Painlevé equations. IV. Third Painlevé equation $\mathrm{P}_{\text {III }}$ Funkcial. Ekvacioj 30 305-332.

Olver, P.J. 1993 "Applications of Lie Groups to Differential Equations," Second Edition, SpringerVerlag, New York.

Rogers, C. 1993 On gasdynamic-solitonic connections in "Applications of Analytic and Geometric Methods to Nonlinear Differential Equations," (Ed. P.A. Clarkson) NATO ASI Series C: Mathematical and Physical Sciences, 413, Kluwer, Dordrecht, pp217-228.

Schief, W.K. 1992 On localized solitonic solutions of a $(2+1)$-dimensional sine-Gordon system $J$. Phys. A: Math. Gen. 25 L1351-L1354.

Schief, W.K. 1994 Bäcklund transformations for the (un)pumped Maxwell-Bloch system and the fifth Painlevé equation J. Phys. A: Math. Gen. 27 547-557.

Weiss, J., Tabor, M. \& Carnevale, G. 1983 The Painlevé property for partial differential equations J. Math. Phys. 24 522-526.

Whittaker, E.E. \& Watson, G.M. 1927 "Modern Analysis," Fourth Edition, C.U.P., Cambridge.

Winternitz, P. 1992 Physical applications of Painlevé type equations quadratic in the highest derivatives in "Painlevé Transcendents, their Asymptotics and Physical Applications," (Eds. 
P. Winternitz \& D. Levi) NATO ASI Series B: Physics, 278, Plenum, New York, pp425-431.

\section{Figure captions}

Figure 1. The solution (3.8a) where $P=\frac{1}{2} \theta(x+y, 2 t), \tilde{P}=\frac{1}{2} \theta(x-y, 2 \tilde{t})$ and $\theta$ is given by (4.4), with $\lambda=1, \mu=1.3$, and $\tilde{t}=-2 t$, for (i) $t=2$, (ii) $t=4$ and (iii) $t=6$.

Figure 2. The solution $(3.8 a)$ where $P=\frac{1}{2} \theta(x+y, 2 t), \tilde{P}=\frac{1}{2} \theta(x-y, 2 \tilde{t})$, and $\theta$ is given by (4.5) with $\beta_{1}=-1, \beta_{2}=2$, and $\tilde{t}=2 t$, for (i) $t=0$, (ii) $t=4$ and (iii) $t=8$.

Figure 3. The solution (3.8a) where $P=\frac{1}{2} \theta(x+y, 2 t), \tilde{P}=\frac{1}{2} \theta(x-y, 2 \tilde{t})$ and $\theta$ is given by (4.4) with $\lambda=1, \mu=1.3, y=5$, but where $x$ has been scaled to $-\exp \left(1-x^{2}\right), t$ has been scaled to $\exp \left(1-t^{2}\right)$ and $\tilde{t}(t)=-\exp \left(1-t^{2}\right)$.

Figure 4. The solutions $(4.31 b, c)$ to the equation (4.10) are plotted in (i) and (ii) respectively, with $\alpha=1$.

Figure 5. The solutions $(4.32 a, b, c)$ to the equation (4.10) are plotted in (i), (ii) and (ii) respectively.

Figure 6. The solution $(4.19 a)$ where $F, \hat{F}$ are both given by $(4.32 c)$, shown for (i) $t=1$, (ii) $t=4$ and (iii) $t=25$.

Figure 7. The solution $(4.19 a)$ where $F$ is given by $(4.32 c)$ and $\hat{F}$ is given by $(4.31 c)$, at $t=1$. 